\title{
An experimental investigation of drop deformation and breakup in steady, two-dimensional linear flows
}

\author{
By B. J. BENTLEY† AND L. G. LEAL \\ Chemical Engineering Department, California Institute of Technology, \\ Pasadena, CA 91125, USA
}

(Received 11 April 1985 and in revised form 21 December 1985)

We consider the deformation and burst of small fluid droplets in steady linear, two-dimensional motions of a second immiscible fluid. Experiments using a computercontrolled, four-roll mill to investigate the effect of flow type are described, and the results compared with predictions of several available asymptotic deformation and burst theories, as well as numerical calculations. The comparison clarifies the range of validity of the theories, and demonstrates that they provide quite adequate predictions over a wide range of viscosity ratio, capillary number, and flow type.

\section{Introduction}

In this paper, we discuss the behaviour of a fluid drop, freely suspended in a second, immiscible, viscous fluid which is undergoing a general linear two-dimensional flow. The flow-induced stress on the drop surface tends to deform the drop, and the interfacial tension between the phases resists this deformation. Under some conditions, the interfacial forces are insufficient to balance the viscous stresses, and the drop bursts. The problem is of both practical and academic interest, and has thus received considerable attention in the fluid-mechanics literature over the past fifty years. Our particular contribution lies in a systematic investigation of the effect of vorticity in the imposed flow for so-called 'strong' flows, where the magnitude of the strain rate exceeds that of the vorticity.

In most practical applications, the objective is to disperse one fluid phase in another, either to form an emulsion, or to increase the surface area between the two phases for more efficient heat and/or mass transfer. In these cases, determination of flow conditions resulting in drop burst is of paramount importance. Examples include dispersion of anti-static or anti-soiling agents, dispersion of colour concentrates, and blending of immiscible polymer systems to form two-phase structures of unique properties (Grace 1971).

Even when the drop does not burst, the distortion produced by a given flow is of interest in understanding the rheological behaviour of flowing emulsions. Emulsions are known to exhibit such non-Newtonian characteristics as shear-dependent viscosity, viscoelasticity, and normal stress differences in rectilinear flow, even when the concentration of the dispersed phase is small (Frankel \& Acrivos 1970; Barthès-Biesel \& Acrivos 1973b). From a knowledge of the deformation of the drops forming the dispersed phase and of the disturbance flow in their vicinity, a constitutive equation can be developed (at least in principle) for the emulsion.

† Current address: Dynamic Solutions, Inc., 2355 Portola Road, Ventura, CA 93003, USA. 
From a theoretical point of view, the drop-deformation problem is extremely difficult. The equations of motion must be solved for the flow both inside and outside the drop, with boundary conditions applied on its surface. However, the shape of the drop is not known a priori, but must be determined as part of the solution. To date, no general solution has been found, but progress has been made through asymptotic analysis for slightly deformed drops, a slender-body theory for highly elongated drops which applies when the viscosity of the drop is small compared with that of the suspending fluid and the velocity gradient is large, and numerical analyses for selected intermediate cases. Recent review articles by Acrivos (1983) and Rallison (1984) describe these efforts in considerable detail. A brief summary is included in $\$ 4$ below.

On the experimental side, a relatively large number of studies of drop deformation and breakup have been reported. These date back to the pioneering work of G. I. Taylor (1934). In this early work, Taylor investigated drop behaviour experimentally in two flow fields, simple shear flow, where the magnitudes of the vorticity and strain rate are equal, and two-dimensional pure-straining flow. The former flow was generated in a parallel-band apparatus, and the four-roll mill was evidently invented to produce the latter. Although few in number, Taylor's experiments uncovered most of the qualitative aspects of the drop deformation and burst process, including the following general conclusions:

1. At low flow strengths, drops of all viscosity ratios deform into prolate spheroids. The longest axis of the drop is initially aligned with the principal axis of strain for both irrotational and simple shear flows.

2. When the drop viscosity is low compared with that of the suspending fluid, the shear rate required for burst becomes quite large, and the drops attain highly deformed steady shapes with pointed ends. Under some conditions, small drops are ejected from these pointed ends, a phenomena which has come to be called 'tip streaming'.

3. When the ratio of drop to suspending fluid viscosity is large, drop behaviour is qualitatively different in simple shear and irrotational flow fields. In irrotational flows, burst occurs at low strain rates. In simple shear, on the other hand, viscous drops assume slightly deformed shapes which are unaffected by further increases in the shear rate, and drop burst becomes impossible beyond a certain critical viscosity ratio.

The dramatic qualitative difference in drop burst between pure-straining flow and simple shear flow furnishes a motivation for study of the drop-deformation and burst process in flows of intermediate vorticity. While such flows can be generated in the four-roll mill (Giesekus 1962; Fuller \& Leal 1981), experimental difficulties in controlling the drop at the centre stagnation point of such flows have, until now, prevented studies of their effect on drop behaviour. In the limiting case when there is no vorticity (the case considered by Taylor 1934), the control problem is simplified because the dividing streamlines are at right angles to the roller geometry, and hand control is possible, though only at the cost of fairly large variations in the flow with time. For other strong flows, however, the dividing streamlines are at angles to the roller geometry, and the complications are too severe for successful manual control. For this reason, almost all of the drop-deformation and burst experiments that followed those of Taylor (1934) (e.g. Rumscheidt \& Mason 1961 ; Torza, Cox \& Mason 1972; Grace 1971) have been restricted to simple shear and/or two-dimensional pure-straining flow. A notable exception is the work of Hakimi \& Schowalter (1980), who studied drop deformation in the flow produced in an orthogonal rheometer. In this device, flows of varying vorticity-to-strain-rate ratio can be generated, but the 
flows are always 'weak', meaning that the magnitude of the vorticity is always larger than that of the strain rate. Thus, the experiments were limited to small deformations $\left(D_{\mathrm{p}} \leqslant 0.2\right)$, and only one viscosity ratio $(\lambda=0.09)$.

In order to investigate experimentally the problem of drop deformation and burst for flows between simple shear and hyperbolic extension, we developed a computerbased control system for the four-roll mill. The drop position was sensed using a digital television camera, and the speeds of stepping motors driving the rollers were regulated to maintain the drop at the centre of the device. A detailed description of the apparatus and control system is reported in Bentley \& Leal (1986). Using this device, we systematically investigated the effect of flow type on the deformation and burst of drops in Newtonian fluids, covering a wide range of viscosity and strainrate-to-vorticity ratios. Computer control of the experiment not only allowed us to study intermediate flows that had not been previously investigated, but also resulted in drop deformation and burst data of considerably improved quality for the irrotational flow limit. In the present paper, we report the results of this experimental investigation, including detailed comparisons with the predictions of available drop deformation and burst theories.

\section{Problem statement}

We consider the behaviour of a drop of volume $\frac{4}{3} \pi a^{3}$, viscosity $\mu^{\prime}$ and density $\rho^{\prime}$, that is freely suspended in an infinite bath of a second fluid of viscosity $\mu$ and density $\rho$. The interfacial tension between the two immiscible fluids is $\sigma$. The interface is assumed to transmit tangential stresses undiminished; thus other possible surface effects such as interfacial viscosity and interfacial tension gradients are neglected. Far from the drop, the suspending fluid undergoes a steady linear flow. The situation is illustrated schematically in figure 1. Both fluids are Newtonian and incompressible, so that the governing equations are the Navier-Stokes equations and the continuity equation, applied inside and outside the drop. At the drop surface, the velocity fields satisfy the conditions of continuity of velocity and tangential stress, and the normal stress suffers a jump due to the interfacial tension. In our experiments in the four-roll mill, we generate an approximation to the idealized linear flow in a bath of finite size. Also, surface impurities present in the real system may affect the behaviour of the fluid-fluid interface. We assume that these are small effects.

When the governing equations are put in dimensionless form, with the undeformed radius of the drop $a$ as the characteristic lengthscale, the inverse of the magnitude of the velocity-gradient tensor, $G^{-1}$, as the characteristic timescale, and $a G$ as the velocity scale, the following dimensionless parameters appear:

$$
\begin{aligned}
& \lambda=\frac{\mu^{\prime}}{\mu} \quad \text { (viscosity ratio), } \\
& \mathbb{C}=\frac{G \mu a}{\sigma} \quad \text { (Capillary number), } \\
& \mathbb{R}=\frac{\rho G^{2} a}{\mu} \quad \text { (Reynolds number), } \\
& \kappa=\frac{\rho^{\prime}}{\rho} \quad \text { (density ratio). }
\end{aligned}
$$

In our experiments, we restricted our attention to cases where viscous effects dominated, so that the Reynolds number based on the drop size was always negligible. 


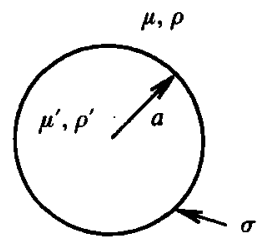

$$
\alpha, G
$$

Fiqure 1. Schematic of problem.

We also considered only neutrally buoyant drops, $\kappa=1$. Under these conditions, the evolution of the drop shape depends only on the viscosity ratio, the capillary number, and the nature of the applied flow. In the flows which can be (approximately) generated in the four-roll mill, the form of the velocity-gradient tensor is characterized by a single parameter $\alpha$, which specifies the relative strength of the strain rate and vorticity in the flow. This parameter is defined by:

$$
\boldsymbol{\nabla} \boldsymbol{u}=\frac{1}{2} G\left[\begin{array}{ccc}
1+\alpha & 1-\alpha & 0 \\
-1+\alpha & -1-\alpha & 0 \\
0= & 0 & 0
\end{array}\right]
$$

The ratio of the magnitude of the rate-of-strain tensor to that of the vorticity in such flows can be expressed as:

$$
\frac{\text { magnitude of strain rate }}{\text { magnitude of vorticity }}=\frac{1+\alpha}{1-\alpha} \text {. }
$$

In particular, $\alpha=+1$ for pure-straining flow, $\alpha=0$ for simple shear flow, and $\alpha=-1$ for purely rotational flow. Streamlines for the positive values of $\alpha$ are shown in figure 2 . For any $\alpha$, the vorticity vector is in the negative $z$-direction, and the principal axes of the rate-of-strain tensor are in the $x$-and $y$-directions. The angle $\theta_{\mathrm{e}}$ between the $x$-axis and the linear exit streamline is given by

$$
\sin \left(2 \theta_{\mathrm{e}}\right)=\frac{\alpha-1}{\alpha+1} \text {. }
$$

In our experiments, photographs of the drop were taken with the camera mounted perpendicular to the plane of the flow, yielding a projection of the drop in the $(x, y)$-plane. For convenient comparisons to theoretical predictions, two distinct scalar measures of drop deformation were determined. These are illustrated in figure 3. The first defines a deformation parameter $D_{\mathrm{f}}$ in terms of the longest and shortest semi-axes of the drop cross-section ( $L$ and $B$ respectively), following Taylor (1934):

$$
D_{\mathrm{f}}=\frac{L-B}{L+B}
$$

and is strictly applicable only for elliptically deformed drops, though it is used in practice whenever the deformation is small. This parameter is zero for spherical drops and asymptotically approaches unity as the drops become infinitely extended. When $L / B$ is large (highly deformed drops), however, $D_{\mathrm{f}}$ changes very little with increasing deformation, and in this case a different measure of the deformation is more appropriate. We follow the precedent of previous studies and choose the ratio of the 


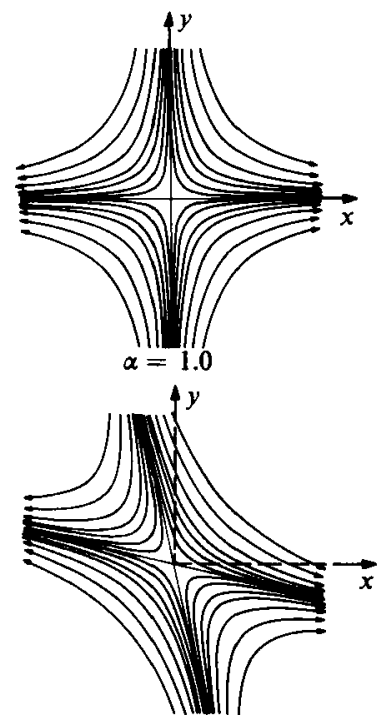

$\alpha=0.4$
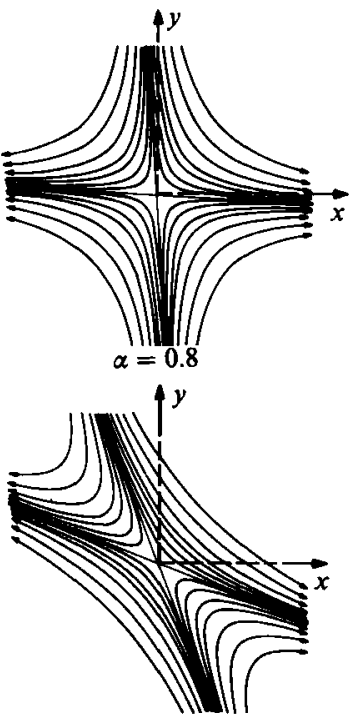

$\alpha=0.2$
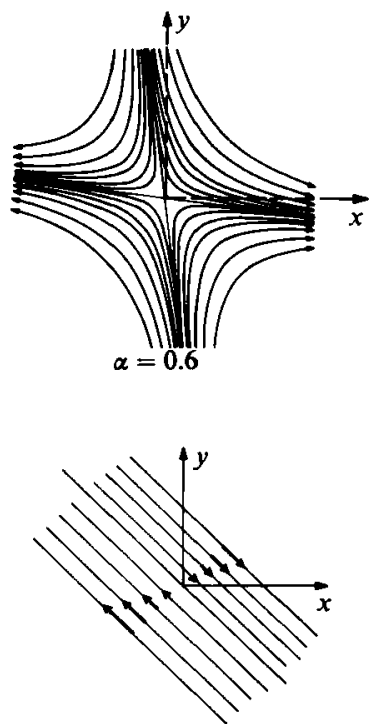

$\alpha=0.0$

Figure 2. Streamlines of flow field of equation (1) for $\alpha \geqslant 0$.

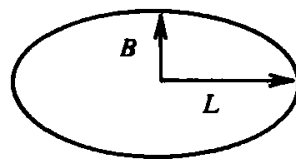

$$
D_{1}=\frac{L-B}{L+B}
$$

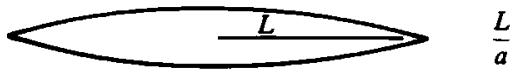

Figure 3. Scalar measurements of deformation.

half-length of the deformed drop to the undeformed radius $L / a$. In all cases, the orientation angle of the drop (the angle between the longest axis of the drop and the major principal axis of the rate-of-strain tensor) was also measured.

In the experiments reported here, we focused our attention on two aspects of drop behaviour. First, we investigated the equilibrium deformation and orientation of drops in steady flows as a function of $C$, for various values of viscosity ratio and flow type:

$$
D_{\mathrm{f}}=D_{\mathrm{f}}(\mathbb{C} ; \lambda, \alpha) \quad \text { or } L / a=L / a(\mathcal{C} ; \lambda, \alpha) \quad \text { and } \theta=\theta(C ; \lambda, \alpha) .
$$

Second, we investigated the critical capillary number required for drop burst, as a function of flow type and viscosity ratio:

$$
\mathbb{C}_{\mathrm{c}}=\mathbb{C}_{\mathrm{e}}(\lambda ; \alpha)
$$

When burst was observed, the maximum stable deformation (the deformation at $\mathbb{C}$ just below $C_{\mathrm{c}}$ ) and corresponding orientation angle were measured:

$$
D_{\mathrm{f}_{\mathrm{c}}}=D_{\mathrm{f}_{\mathrm{c}}}(\lambda ; \alpha) \text { and } \theta_{\mathrm{c}}=\theta_{\mathrm{c}}(\lambda ; \alpha)
$$




\section{Fluid systems and experimental procedure}

The four-roll mill used in this study, and the associated video camera/computer control system, are described in detail by Bentley (1985), and by Bentley \& Leal (1986). For present purposes, we note that the cylinders had a diameter of $10.16 \mathrm{~cm}$ and a length of $15.5 \mathrm{~cm}$, and that the gap width between adjacent rollers was set at $2.54 \mathrm{~cm}$. In general, it has been shown that a good approximation to a linear flow, i.e. $\boldsymbol{u}=\boldsymbol{\nabla} \boldsymbol{u} \cdot \boldsymbol{x}$, with $\boldsymbol{\nabla} \boldsymbol{u}$ given by (5), can be realized in a well-designed four-roll mill over a square central region between rollers whose sides are approximately equal to the gap width between rollers, i.e. $2.54 \mathrm{~cm}$ in the present case, provided that $|\alpha| \geqslant 0.2$. Thus, the dimensions of our four-roll mill are sufficient to allow a drop with an undeformed diameter of $1 \mathrm{~mm}$ (typical of our experiments) to be stretched to a length 25 times its initial radius without encountering any significant deviation from the ideal linear flow for these flow types. The restriction to values $|\alpha| \geqslant 0.2$ is a consequence of geometric constraints which restrict the accuracy with which flows with smaller $\alpha$ can be generated in the four-roll mill. For example, simple shear flow (i.e. $\alpha=0$ ) is achieved (approximately) as the limit when one diagonally opposing pair of rollers is co-rotating, while the other pair is completely stationary. But the second, stationary pair of rollers lies directly in the path of the flow, and this disrupts the motion and degrades the comparison between the ideal simple shear flow and the flow which is actually produced in the four-roll mill.

Drop deformation and burst experiments were performed for fourteen different drop-suspending fluid systems, ranging in viscosity ratio from 0.001 to about 100 . The drop fluids were a series of eleven Dow Corning Silicon fluids, with kinematic viscosities ranging from 5 to 60000 cst. Two suspending fluids, Pale 4 Oil and Pale 170 Oil (oxidized castor oils available from CasChem, Inc. of Bayonne, NJ) were used. Table 1 shows the measured viscosity and density for each of these fluids. For each fluid system, five different flow types, $\alpha=1.0,0.8,0.6,0.4$ and 0.2 , were investigated.

The kinematic viscosities and densities of each fluid were measured as a function of temperature in the range of $20-25^{\circ} \mathrm{C}$ using a series of Cannon-Fenske capillary viscometers and a pycnometer. All of the fluids have been reported to be Newtonian over the shear-rate range covered by our experiments (Rumscheidt \& Mason 1961). The interfacial tension between the fluids was measured using a ring tensiometer (Fisher Scientific Model 20). These interfacial tension measurements were generally not very accurate owing to the small density differences between the two phases. Consequently, the interfacial tension was also calculated from the initial slope of $D_{\mathrm{p}}$ versus $G \mu a$, assuming that the $O(1)$ theory of Taylor (1934) accurately describes the drop deformation in the limit of small deformations. Table 2 shows a comparison of the interfacial tensions measured from the ring-tensiometer experiments and inferred from the drop deformation experiments, and figure 4 shows a graphical comparison of the two. As can be seen, the two measures were generally in reasonable agreement, with an average difference of $3.3 \%$ and a maximum difference of $14.2 \%$ between them. The ring-tensiometer values were consistently lower than those inferred from the drop deformation data, perhaps indicating some aging of the interface in this measurement which is absent in the deformation experiments. A more pronounced effect of this type was reported by Grace (1971). Because they are thought to be more reliable, the interfacial tensions computed from the small deformation theory were used in both the deformation and burst plots in this paper.

For each fluid-system/flow-type combination, the following experimental procedure was employed: A small drop ( $\approx 1 \mathrm{~mm}$ radius) was introduced into the centre of the four-roll mill. The computer was directed to centre the drop (by turning the 


\begin{tabular}{|c|c|c|c|c|c|c|c|}
\hline \multirow{2}{*}{$\begin{array}{c}\text { Fluid } \\
\text { no. }\end{array}$} & \multirow[b]{2}{*}{ Fluid type } & \multicolumn{3}{|c|}{ Viscosity (P) } & \multicolumn{3}{|c|}{ Density $\left(\mathrm{g} / \mathrm{cm}^{3}\right)$} \\
\hline & & $20.0^{\circ}$ & $22.5^{\circ}$ & $25.0^{\circ}$ & $20.0^{\circ}$ & $22.5^{\circ}$ & $25.0^{\circ}$ \\
\hline 1 & Pale 4 oil & 53.32 & 41.97 & 33.55 & 0.9961 & 0.9953 & 0.9931 \\
\hline 2 & Pale 170 oil & 14.17 & 11.44 & 9.475 & 0.9773 & 0.9756 & 0.9736 \\
\hline 3 & $5 \mathrm{cs} \mathrm{SF}$ & 0.0519 & 0.0495 & 0.0474 & 0.9181 & 0.9161 & 0.9138 \\
\hline 4 & $50 \mathrm{cs} \mathrm{SF}$ & 0.5273 & 0.5008 & 0.4774 & 0.9623 & 0.9602 & 0.9578 \\
\hline 5 & 100 cs SF & 1.050 & 0.9964 & 0.9483 & 0.9645 & 0.9618 & 0.9598 \\
\hline 6 & $200 \mathrm{cs} \mathrm{SF}$ & 2.139 & 2.031 & 1.937 & 0.9692 & 0.9667 & 0.9657 \\
\hline 7 & $500 \mathrm{cs} \mathrm{SF}$ & 5.336 & 5.059 & 4.807 & 0.9721 & 0.9697 & 0.9676 \\
\hline 8 & 1000 es SF & 11.07 & 10.56 & 10.02 & 0.9716 & 0.9697 & 0.9675 \\
\hline 9 & 2000 cs SB & 27.76 & 25.69 & 24.41 & 0.9732 & 0.9718 & 0.9696 \\
\hline 10 & 5000 cs SB & 70.21 & 66.88 & 63.46 & 0.9737 & 0.9721 & 0.9699 \\
\hline 11 & 10000 cs $\mathrm{SB}$ & 126.1 & 119.1 & 113.0 & 0.9747 & 0.9724 & 0.9703 \\
\hline 12 & 30000 es SF & 320.6 & 303.2 & 289.0 & 0.9754 & 0.9729 & 0.9707 \\
\hline 13 & $60000 \operatorname{cs~SF}$ & 673.3 & 638.8 & 607.7 & 0.9777 & 0.9751 & 0.9726 \\
\hline 14 & $1000000 \mathrm{cs} \mathrm{SF}$ & 1082.5 & 1018.8 & 968.8 & 0.9878 & 0.9782 & 0.9735 \\
\hline
\end{tabular}

TABLE 1. Viscosity and density as a function of temperature for the fluids used in this study. The notation SF indicates a commercially available grade of silicon fluid, while SB indicates a blend of two grades resulting in the specified properties.

\begin{tabular}{|c|c|c|c|c|c|c|c|c|}
\hline $\begin{array}{c}\text { System } \\
\text { no. }\end{array}$ & $\begin{array}{l}\text { Susp. } \\
\text { fluid }\end{array}$ & $\begin{array}{l}\text { Drop } \\
\text { fluid }\end{array}$ & $\underset{{ }^{\circ} \mathrm{C}}{\text { Temp. }}$ & $\begin{array}{l}\mu_{\mathrm{c}} \\
(\mathrm{P})\end{array}$ & $\begin{array}{l}\mu_{\mathrm{d}} \\
(\mathrm{P})\end{array}$ & $\underset{\lambda}{\sigma_{\mathrm{RT}}}$ & $\underset{\text { (dynes/cm) }}{\sigma_{\mathrm{SD}}}$ & (dynes/cm) \\
\hline 1 & 1 & 3 & 20.8 & 49.5 & 0.051 & 0.001 & 3.44 & 3.98 \\
\hline 2 & 1 & 4 & 21.6 & 45.511 & 0.011 & 5.34 & 5.32 & - \\
\hline 3 & 1 & 5 & 21.6 & 46.45 & 1.015 & 0.22 & 4.55 & 5.23 \\
\hline 4 & 1 & 6 & 22.3 & 42.80 & 2.038 & 0.048 & 4.52 & 5.27 \\
\hline 5 & 1 & 7 & 22.5 & 42.00 & 5.059 & 0.12 & 5.25 & 5.54 \\
\hline 6 & 1 & 8 & 21.8 & 45.25 & 10.70 & 0.24 & 5.14 & 5.51 \\
\hline 7 & 1 & 9 & 22.8 & 40.80 & 25.52 & 0.63 & 5.34 & 5.37 \\
\hline 8 & 1 & 10 & 21.9 & 44.60 & 67.68 & 1.52 & 5.41 & 5.41 \\
\hline 9 & 1 & 11 & 22.4 & 42.45 & 119.3 & 2.81 & 5.40 & 5.46 \\
\hline 10 & 1 & 12 & 21.2 & 47.8 & 313.0 & 6.55 & 5.52 & 5.98 \\
\hline 11 & 1 & 13 & 21.8 & 45.25 & 648.6 & 14.3 & 5.66 & 5.94 \\
\hline 12 & 2 & 12 & 22.0 & 11.93 & 308.0 & 25.8 & - & 5.19 \\
\hline 13 & 2 & 13 & 22.7 & 11.25 & 637.3 & 56.6 & - & 5.53 \\
\hline 14 & 2 & 14 & 22.4 & 11.53 & 1021.0 & 88.6 & - & 5.41 \\
\hline
\end{tabular}

TABLE 2. Fluid system properties, corresponding to the conditions of the experiments. The fluid numbers refer to table 1. $\sigma_{\mathrm{RT}}$ indicates the interfacial tension measured using the ring-tensiometer method, and $\sigma_{\mathrm{SD}}$ indicates the interfacial tension inferred from the small-deformation data.

appropriate rollers). A still photograph of the undeformed drop was taken for later determination of its exact size. The temperature of the suspending fluid was measured to allow interpolation of the viscosity of the two phases.

At this point, the flow was started at the lowest practical shear rate (about $0.05 \mathrm{~s}^{-1}$ ). After a period of time which depended on the viscosity ratio (longer for higher viscosity ratios), the drop was assumed to be at an equilibrium deformation and a photograph taken. For convenience, the computer was directed to log the shear rate, time and picture number. The shear rate was then slowly increased through a series of small steps (about $0.01 \mathrm{~s}^{-1}$ each). Photographs were taken at intervals in the shear rate sufficiently closely spaced to define the deformation curve accurately. 


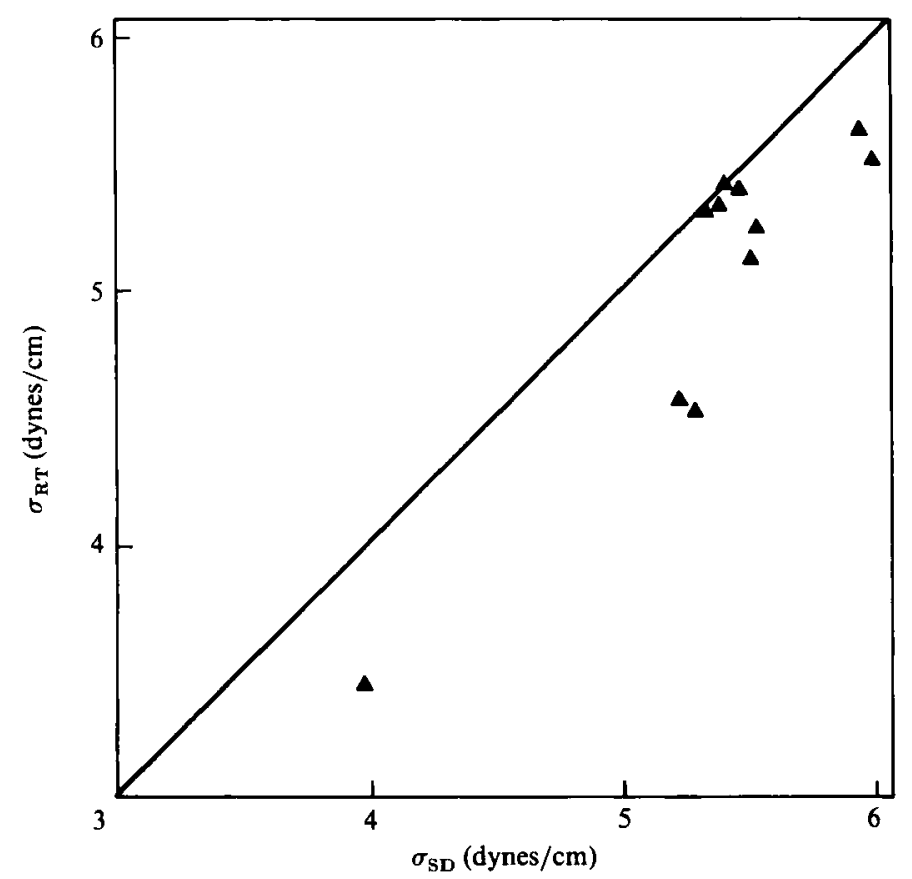

Figure 4. Comparison of ring tensiometer and drop deformation interfacial tension measures.

In most experiments, a shear rate was reached for which no steady drop shape was possible. This was termed the critical shear rate for drop burst. When this point was reached, the drops were allowed to extend until their length was approximately ten times their initial diameter, and then the flow was turned off. The drops did not fragment while the flow was on, but when it was stopped, most of the drops broke up through a complex, interfacial-tension-driven motion. Some of the highestviscosity-ratio drops returned to the initial spherical shape without fragmenting. The transient motion of the drops in the critical flow and the motion subsequent to stopping the external flow will be the subject of a future communication.

In some cases (high-viscosity-ratio drops in the more rotational flow fields) the drops did not burst with increasing shear rate, but instead attained a small deformation which remained essentially constant with increasing shear rate. This is analogous to the behaviour in simple shear.

The photographs from the experiments were analysed by projecting the negatives onto the screen of a microfiche viewer. The length, breadth, and orientation angle of each drop was measured, allowing calculation of the scalar deformation measures $D_{\mathrm{f}}$ and $L / a$.

\section{Theoretical estimates of drop deformation and conditions for burst}

We have mentioned previously that there have been several theoretical studies of drop deformation and burst. One of the main objectives of our present investigation is to provide a detailed comparison with the predictions from these theories for the drop shape and the conditions for burst. In this section, we review the existing theoretical analyses, particularly those aspects that are necessary for providing predictions of drop shapes for the specific conditions and flows that we have studied experimentally. 


\subsection{Small-deformation theory}

We begin with the small-deformation theory for zero Reynolds number that was pioneered by Taylor $(1932,1934)$. In this approach it is assumed that conditions exist such that the drop is only slightly deformed from spherical, so that the method of domain perturbations can be used to determine the velocity fields inside and outside the drop, as well as the drop shape. Higher-order corrections to Taylor's original analysis have been considered by a number of authors, including Cox (1969) and Barthès-Biesel \& Acrivos (1973a). The results of these investigations were unified and clarified in a critical review by Rallison (1980).

Rallison (1980) presented a general analysis, valid for nearly spherical inertialess drops in a linear shear field. In this formulation, the drop surface $r$ is represented by a superposition of spherical harmonics of second and fourth order:

$$
r=1+\epsilon r^{3} \boldsymbol{F}: \nabla \nabla\left(\frac{1}{r}\right)+\epsilon^{2}\left[\left(-\frac{6}{5}\right) \boldsymbol{F}: \boldsymbol{F}+r^{5} \boldsymbol{H}: \nabla \nabla \nabla \nabla\left(\frac{1}{r}\right)\right]+O\left(\epsilon^{2}\right) .
$$

Here $r=(x \cdot x)^{\frac{1}{2}}$, and the dimensions are scaled by $a$, the undeformed radius of the drop. The tensors $\boldsymbol{F}$ and $\boldsymbol{H}$ are symmetric and traceless tensors of second and fourth rank respectively. Rallison (1980) gives equations for the evolution of these tensors as:

$$
\begin{aligned}
& \epsilon \frac{\mathrm{D} \boldsymbol{F}}{\mathrm{D} t}=a_{0} \boldsymbol{E}+\epsilon\left\{\mathbb{C}^{-1} a_{1} \boldsymbol{F}+a_{2} \operatorname{Sd}(\boldsymbol{E} \cdot \boldsymbol{F})\right\}+\epsilon^{2}\left\{\boldsymbol{C}^{-1} a_{3} \operatorname{Sd}(\boldsymbol{F} \cdot \boldsymbol{F})\right. \\
& \left.+a_{4} E \boldsymbol{F}: \boldsymbol{F}+a_{5} \boldsymbol{F E}: \boldsymbol{F}+a_{7} \operatorname{Sd}(\boldsymbol{E} \cdot \boldsymbol{F} \cdot \boldsymbol{F})+a_{8} \boldsymbol{H}: \boldsymbol{E}\right\}+O\left(\epsilon^{3}, C^{-1} \epsilon^{3}\right) \\
& \epsilon \frac{\mathrm{D} \boldsymbol{H}}{\mathrm{D} t}=b_{1} \operatorname{Sd}_{4}(\boldsymbol{E} \boldsymbol{F})+\epsilon\left\{b_{0} C^{-1} \boldsymbol{H}+b_{2} C^{-1} \operatorname{Sd}_{4}(\boldsymbol{F} \boldsymbol{F})+b_{3} \operatorname{Sd}_{4}(\boldsymbol{E} \cdot \boldsymbol{H})\right. \\
& \left.+b_{4} \operatorname{Sd}_{4}(\boldsymbol{E} \cdot \boldsymbol{F} \boldsymbol{F})\right\}+O\left(\epsilon^{2}, \boldsymbol{C}^{-1} \epsilon^{2}\right),
\end{aligned}
$$

where $E$ is the rate-of-strain tensor. The vorticity enters through the Jaumann, or corotational, derivative which is defined for a second-order tensor, $\boldsymbol{A}$, as:

$$
\frac{\mathrm{D} \boldsymbol{A}}{\mathrm{D} t}=\frac{\partial \boldsymbol{A}}{\partial t}+\boldsymbol{v} \cdot \boldsymbol{\nabla} \boldsymbol{A}+(\boldsymbol{\Omega} \cdot \boldsymbol{A}-\boldsymbol{A} \cdot \boldsymbol{\Omega})
$$

where $\Omega$ is the vorticity tensor. In (13) and (14), $a_{0}-a_{8}$ and $b_{0}-b_{4}$ are rational functions of the viscosity ratio, and all except $b_{3}$ and $b_{4}$, which have not been derived to date, are given by Barthès-Biesel \& Acrivos (1973a). The definitions of the symmetric deviators of second- and fourth-rank tensors, $\mathrm{Sd}$ and $\mathrm{Sd}_{4}$, are also supplied. Retaining only the first two terms on the right-hand side of (13) yields Taylor's original result.

These equations apply whenever the deviation from sphericity is small. Steady small deformations occur in two distinct cases; either the flow strength is weak (C small), or the viscosity ratio is large in a flow with vorticity. Barthès-Biesel \& Acrivos (1973a) applied (12)-(14) for the weak-flow case, setting $\epsilon=\mathbb{C}$ in (13), and including the terms of order $\epsilon^{3} C^{-1}$ (the $a_{6}$ and $a_{9}$ terms which appear in (16)). They obtained the equations:

$$
\begin{aligned}
\epsilon \frac{\mathrm{D} \boldsymbol{F}}{\mathrm{D} t}=a_{0} \boldsymbol{E}+a_{1} \boldsymbol{F}+\epsilon\left\{a_{2} \operatorname{Sd}(\boldsymbol{E} \cdot \boldsymbol{F})\right. & \left.+a_{3} \operatorname{Sd}(\boldsymbol{F} \cdot \boldsymbol{F})\right\}+\epsilon^{2}\left\{a_{4} \boldsymbol{E} \boldsymbol{F}: \boldsymbol{F}+a_{5} \boldsymbol{F}: \boldsymbol{F}+a_{6} \boldsymbol{F} \boldsymbol{F}: \boldsymbol{F}\right. \\
& \left.+a_{7} \operatorname{Sd}(\boldsymbol{E} \cdot \boldsymbol{F} \cdot \boldsymbol{F})+a_{8} \boldsymbol{H}: \boldsymbol{E}+a_{9} \boldsymbol{H}: \boldsymbol{F}\right\}+O\left(\epsilon^{3}\right)
\end{aligned}
$$


There are several important points concerning the expansion leading to (16) and (17). First, as noted by Bathès-Biesel \& Acrivos (1973a), algebraic complexities forced some inconsistencies in the order of expansion. From (16), the second-order deformation tensor $\boldsymbol{F}$ is known to $O\left(\epsilon^{2}\right)$. For consistency in calculation of the shape (equation (12)) the fourth-order tensor $H$ should thus be known to $O(\epsilon)$. However, the coefficients $b_{3}$ and $b_{4}$ could not be calculated, so (14) was necessarily truncated after the $O(1)$ terms. Similarly, terms of $O\left(\epsilon^{3}\right)$, including a sixth-rank deformation tensor, should be included in (12), but these also could not be derived. Barthès-Biesel \& Acrivos (1973a) suggest using (12), (16), and (17) as an approximation to the full asymptotic problem, and that is the approach followed here, with the exception discussed below for high-viscosity-ratio cases.

When the small deformation is due to a high viscosity ratio in a rotational flow instead of a small capillary number, a different expansion is more appropriate, in which $1 / \lambda$ appears as the small parameter. In this case, the behaviour of the coefficients $a_{i}$ and $b_{i}$ as $\lambda \rightarrow \infty$ cause some of the terms in (13) and (14) to 'jump order'. In the high- $\lambda$ limit, (13) and (14) yield (Rallison 1980 equations (8) and (9)):

$$
\begin{aligned}
& \frac{\mathrm{D} \boldsymbol{F}}{\mathrm{D} t}=\frac{5}{6} \boldsymbol{E}+\lambda^{-1}\left\{-\frac{20}{19} C^{-1} \boldsymbol{F}+\right. \frac{10}{7} \operatorname{Sd}(\boldsymbol{E} \cdot \boldsymbol{F})-\frac{5}{4} \boldsymbol{E}-3 \boldsymbol{E} \boldsymbol{F}: \boldsymbol{F} \\
&+18 \mathrm{Sd}(\boldsymbol{E} \cdot \boldsymbol{F} \cdot \boldsymbol{F})-6 \boldsymbol{F} \boldsymbol{E}: \boldsymbol{F}\}+O\left(\lambda^{-2}, C^{-1} \lambda^{-2}\right), \\
& \frac{\mathrm{D} \boldsymbol{H}}{\mathrm{D} t}=\frac{1}{14} \mathrm{Sd}_{4}(\boldsymbol{E} \boldsymbol{F})+b_{3}^{\prime} \operatorname{Sd}_{4}(\boldsymbol{E} \cdot \boldsymbol{H})+b_{4}^{\prime} \operatorname{Sd}_{4}(\boldsymbol{E} \cdot \boldsymbol{F} \boldsymbol{F})+O\left(\lambda^{-1}, C^{-1} \lambda^{-1}\right) .
\end{aligned}
$$

When terms of $O\left(\epsilon^{2}\right)$ are retained in (16), and the limiting values of $a_{i}$ as $\lambda \rightarrow \infty$ substituted, all terms of (18) are preserved (as well as a few which are higher order in $\lambda^{-1}$ and have been truncated from (18)). Therefore, computations using (16) will remain accurate to $O\left(\lambda^{-1}\right)$ for large viscosity ratio. However, owing to the inconsistency in the expansion order mentioned above, (17) does not allow calcuation of $\boldsymbol{H}$ even to $O(1)$ in the high-viscosity-ratio limit. The unknown $b_{3}$ and $b_{4}$ terms in (14), which had to be truncated in going to (17), may 'jump order' (depending on their behaviour as $\lambda \rightarrow \infty$ ) and thus appear as $O(1)$ in (19). Empirically, calculations retaining the fourth-order tensor terms calculated from (17) gave drops with unrealistic lobed shapes for high-viscosity-ratio systems. Therefore, for viscosity ratios greater than 3.0, the fourth-order tensor terms were dropped in calculating shapes from (12). This gave much more realistic drop shapes in the high $\lambda$ limit, and deformations which compared well with the experimental observations.

For the steady-deformation case, simplifications of (16) and (17) are possible. Equation (17) can be solved for $\boldsymbol{H}$ directly in terms of $\boldsymbol{E}$ and $\boldsymbol{F}$, and the result substituted into (16). Use of an identity for the fourth-order symmetric deviator then yields (Barthès-Biesel \& Acrivos 1973a):

$$
\begin{aligned}
0= & a_{0} \boldsymbol{E}+a_{1} \boldsymbol{F}+\epsilon\left\{a_{2} \operatorname{Sd}(\boldsymbol{E} \cdot \boldsymbol{F})+a_{3} \operatorname{Sd}(\boldsymbol{F} \cdot \boldsymbol{F})-\boldsymbol{\Omega} \cdot \boldsymbol{F}+\boldsymbol{F} \cdot \boldsymbol{\Omega}\right\} \\
& +\epsilon^{2}\left\{c_{1} \boldsymbol{E}: \boldsymbol{F}+c_{2} E \boldsymbol{E}: \boldsymbol{F}+c_{3} \boldsymbol{F} \boldsymbol{F}: \boldsymbol{F}+c_{4} \boldsymbol{F}: \boldsymbol{F}+c_{5} \boldsymbol{F}: \boldsymbol{E}\right. \\
& \left.+c_{6} \operatorname{Sd}(\boldsymbol{E} \cdot \boldsymbol{F} \cdot \boldsymbol{F})+c_{7} \operatorname{Sd}(\boldsymbol{E} \cdot \boldsymbol{E} \cdot \boldsymbol{F})\right\},
\end{aligned}
$$

where $c_{1}-c_{7}$ are algebraic combinations of $a_{i}$ and $b_{i}$, and are given by Barthès-Biesel \& Acrivos (1973a)

To apply (12), (17) and (20) to the four-roll-mill flow of (5), $E$ and $\Omega$ are substituted into (20), and the component equations written. The requirements of symmetry and tracelessness yield three independent components of $F ; F_{11}, F_{12}$, and $F_{22}$. For 
convenience, the first two are written in the linear combinations $S=F_{11}+F_{22}$ and $D=F_{11}-F_{22}$. $H$ has five independent non-zero components; $H_{1111}, H_{1112}, H_{1122}, H_{1222}$, and $H_{2222}$. In terms of $S, D$, and $F_{12},(20)$ reduces to the system of nonlinear algebraic equations:

$$
\begin{array}{r}
\begin{aligned}
\begin{array}{r}
0 a_{0} A+ \\
+
\end{array} & a_{1} D+\epsilon\left\{a_{2} A S+a_{3} S D+2(1-\alpha)\right\}+\epsilon^{2}\left\{2 c_{1} A T+2 c_{2} A^{2} D\right. \\
& \left.+c_{3} T D+c_{4} A D^{2}+2 c_{5} A^{2} D+c_{6} A\left(\frac{1}{2} S^{2}+2 F_{12}^{2}+\frac{1}{2} D^{2}\right)+c_{7} A^{2} D\right\}
\end{aligned} \\
\begin{aligned}
0=a_{1} F_{12}+\epsilon & \left\{a_{3} F_{12} S-\frac{1-\alpha}{2} D\right\}+\epsilon^{2}\left\{c_{3} T F_{12}+c_{4} A D F_{12}\right. \\
& \left.+2 c_{5} A^{2} F_{12}+c_{7} A^{2} F_{12}\right\}
\end{aligned} \\
\begin{aligned}
0= & a_{1} S+\epsilon\left\{\frac{a_{2} A D}{3}-a_{3}\left(S^{2}-\frac{1}{3} T\right)\right\}+\epsilon^{2}\left\{c_{3} S T+c_{4} A S D\right. \\
& \left.+2 c_{5} A^{2} S+\frac{c_{6} A S D}{3}+\frac{c_{7} A^{2} S}{3}\right\}
\end{aligned}
\end{array}
$$

where $T$ is defined as $F: F$ and $A$ as $\frac{1}{2}(1+\alpha)$. Substituting into (17) yields:

$$
\begin{aligned}
& H_{1111}=-\frac{b_{1} A}{35 b_{0}}\left(51 F_{11}-6 F_{22}\right)-\frac{b_{2}}{35 b_{0}}\left(57 F_{11}^{2}-48 F_{12}^{2}+12 F_{22}^{2}+12 F_{11} F_{22}\right) \\
& H_{1112}=-\frac{3 b_{1} A}{2 b_{0}} F_{12}-\frac{b_{2}}{7 b_{0}}\left(15 F_{11} F_{12}-6 F_{12} F_{22}\right) \\
& H_{1122}=\frac{b_{1} A}{70 b_{0}}\left(51 F_{11}-51 F_{22}\right)-\frac{b_{2}}{35 b_{0}}\left(-6 F_{11}^{2}+54 F_{12}^{2}-6 F_{22}^{2}+39 F_{11} F_{12}\right) \\
& H_{1222}=\frac{3 b_{1} A}{2 b_{0}} F_{12}+\frac{b_{2}}{7 b_{0}}\left(6 F_{11} F_{12}-15 F_{12} F_{22}\right) \\
& H_{2222}=-\frac{b_{1} A}{35 b_{0}}\left(6 F_{11}-51 F_{22}\right)-\frac{b_{2}}{35 b_{0}}\left(12 F_{11}^{2}-48 F_{12}^{2}+57 F_{22}^{2}+12 F_{11} F_{22}\right)
\end{aligned}
$$

These equations reduce properly to equations (4.1) of Barthès-Biesel \& Acrivos $(1973 a)$ for two-dimensional extensional flow $(\alpha=1)$ and, with appropriate rotation of the coordinate system, to equations (5.1) for simple shear flow $(\alpha=0)$, except that the coefficient of the $c_{7}$ term in equation (5.1) of Barthès-Biesel \& Acrivos (1973a) should be $\frac{1}{3}$ rather than $\frac{1}{6}$.

The system (21)-(23) was solved for $S, D$, and $F_{12}$ using a Newton iteration scheme. Once $S, D$, and $F_{12}$ are known, calculation of the components of $\boldsymbol{F}$ and $\boldsymbol{H}$ is straightforward. For purposes of computing $D_{\mathrm{f}}$, the scalar measure of deformation defined by (8), (12) can be applied in the $(z=0)$-plane, yielding:

$$
\begin{aligned}
r(\theta)=1 & +3 \epsilon\left\{F_{11} \cos ^{2} \theta+2 F_{12} \cos \theta \sin \theta+F_{22} \sin ^{2} \theta\right\} \\
& +\epsilon^{2}\left\{\frac{-6}{5} T+105\left(H_{111} \cos ^{4} \theta+4 H_{1112} \cos ^{3} \theta \sin \theta\right.\right. \\
& \left.\left.+6 H_{1122} \cos ^{2} \theta \sin ^{2} \theta+4 H_{1222} \cos \theta \sin ^{3} \theta+H_{2222} \sin ^{4} \theta\right)\right\},
\end{aligned}
$$

where $\theta$ is the angle from the $x$-axis in the $(x, y)$-plane. The maximum and minimum of $r(\theta)$ are then $L$ and $B$ respectively. The orientation angle is defined as the angle for which $r(\theta)$ is maximum.

The stability of the calculated shapes was assessed through a conventional 
linear-stability analysis as described by Barthès-Biesel \& Acrivos (1973a). For the flow field of $(5)$, the problem is reduced to finding the eigenvalues of two matrices, an $8 \times 8$ matrix of disturbances to the components of $\boldsymbol{F}$ and $\boldsymbol{H}$ which are non-zero at steady state, and a $6 \times 6$ matrix of disturbances to the components which are zero at steady state. The components of the two matrices can be found in Bentley (1985). The eigenvalues were computed using a routine supplied as part of the IMSL subroutine library.

The deformation and orientation curves ( $D_{\mathrm{f}}$ and $\theta$ versus $\epsilon$ or $\mathbb{C}$ ) were calculated for every flow-type/viscosity ratio combination investigated experimentally. Following Barthès-Biesel \& Acrivos (1973a), we calculated the curves to $O(\epsilon)$ by discarding the $O\left(\epsilon^{2}\right)$ terms in (16), and to $O\left(\epsilon^{2}\right)$ by retaining these terms. In both cases, (12) was used in its entirety except that the fourth-order tensor terms were disregarded for $\lambda>3$ as described above. Each curve was calculated by starting at nearly zero $\epsilon$ (or $\mathbb{C}$ ), using an undeformed sphere as the initial guess for the Newton iteration technique. The calculated deformation was used as the new initial guess, and the process repeated for higher $\epsilon$.

In this manner, the deformation curve was computed until some condition indicating drop burst was reached. The calculation was then backed up a step, and the step size reduced by a factor of 20 to determine the critical Capillary number, $\mathbb{C}_{\mathrm{c}}$ (or $\epsilon_{\mathrm{c}}$ ) as closely as possible. We considered burst to be indicated either by the lack of a solution to (21)-(23), or an instability in the solution revealed by the linear stability analysis. In most cases, burst was manifested by inability to find a steady-state solution. When unstable solutions were found, the instability was always in the $8 \times 8$ matrix, and continuing the calculation to higher $\epsilon$ revealed that the value of $\epsilon$ for which no solution existed was within $0.6 \%$ of the value for unstable solutions. Also, when burst was indicated by the lack of existence of a steady solution, the eigenvalues for the $8 \times 8$ matrix were small negative numbers, decreasing in magnitude with increasing $\epsilon$. Thus, the two criteria for burst nearly coincided for the cases we investigated.

It should be noted that, a priori, there is no reason to be particularly optimistic about the accuracy of drop-burst predictions from the small-deformation theory. In general, drop burst occurs at deformations which are not especially small, outside of the range where the small-deformation theory is technically valid. Even disregarding this, there is no rigorous justification for the inherent assumption that the lack of a stable solution to the approximate governing equations necessarily means that a solution of the exact equations is likewise lacking. Thus the burst predictions of the small-deformation theory must be tested, either through experiments or through more exact numerical calculations. As we will see in $\$ 5$, it turns out that the small-deformation theory not only reproduces the qualitative features of the dropburst curves quite accurately, it also provides a surprisingly good quantitative estimate for drop burst when the viscosity ratio is greater than about 0.05 .

\subsection{Large-deformation theory}

The small-deformation theory does not accurately predict drop deformation or burst for $\lambda$ less than about $\mathbf{0 . 0 5}$. For these cases, highly-deformed steady drop shapes are possible, and a theory valid for small perturbations from the spherical shape would not be expected to apply. Fortunately, for $\lambda \ll 1$, an analytical solution which takes advantage of the observed slenderness of the drops is available.

As for the small-deformation theory, Taylor (1964) was the first to present solutions for highly elongated drops. The analysis was refined and clarified by Buckmaster (1972, 1973), and Acrivos \& Lo (1978). 
We consider the analysis for a drop of low, but finite viscosity ratio. This case was considered by Acrivos \& Lo (1978) for an axisymmetric extensional flow

$$
u_{x}=G x, \quad u_{r}=-\frac{1}{2} G r, \quad u_{\theta}=0,
$$

far from the drop. In this case, the drop cross-section is circular and the centreline position is known (aligned with the $x$-axis). The drop is assumed slender, and its surface is represented by:

$$
r=\epsilon R(x) \text {, }
$$

where the slenderness ratio defined by

$$
\epsilon=\frac{\sigma}{G \mu L},
$$

is assumed small. In this problem, the creeping-flow equations must be solved for the flow both inside and outside the drop. The analysis reveals that slender drops can exist only for $\lambda \leqslant O\left(\epsilon^{2}\right)$, and the drop shape is then given by:

$$
R(x)=\frac{1}{8}\left[1+\left(1-\frac{64}{K^{2}}\right)^{\frac{1}{2}}\right]\left(1-x^{2}\right)
$$

where $K^{2} \equiv \epsilon^{2} / \lambda$. Acrivos \& Lo (1978) have shown that this solution is stable only for $K^{2} \leqslant \frac{576}{5}$. When the condition of volume conservation is applied to (33), we obtain the deformation relation:

$$
C \lambda^{\frac{1}{6}}=\left(\frac{1}{20}\right)^{\frac{1}{2}} \frac{\xi^{\frac{1}{2}}}{1+\frac{4}{5} \xi^{3}} .
$$

This equation implicitly relates the dimensionless length, $\xi \equiv L \lambda^{\frac{1}{3}} / a$, to the flow strength. A plot of the deformation curve (34) is given by Acrivos \& Lo (1978). For values of $C \lambda^{\frac{1}{8}}$ up to about 0.12 , the deformation increases rather slowly. At that point, however, the deformation increases sharply, reaching a maximum steady extension of $\xi=0.630$ at $K^{2}=\frac{576}{5}$. This point, defined as drop burst, occurs at a dimensionless shear rate, $C \lambda^{\frac{1}{6}}$ equal to 0.148 . For this burst criterion to be applicable, the slenderness ratio, $R(0) / L$ must be small, which requires that $(5 \lambda)^{\frac{1}{2}} \ll 1$.

Hinch \& Acrivos (1979) extended the slender-drop theory to the case where the applied shear was a two-dimensional straining motion $(\alpha=1$ in (5)), considering this flow field to be a perturbation to axisymmetric straining flow. While the analysis was complicated by the fact that the non-axisymmetric nature of the imposed flow caused the cross-section of the drop to be non-circular, the analysis of Hinch \& Acrivos (1980) revealed that the deformation pattern and burst criterion for drops in the twodimensional flow is nearly identical to that for axisymmetric extensional flow. This is because the cross-sectional area of the drops is very similar in the two flow fields despite differences in the details of the cross-sectional shape, and it is apparently this area which governs the deformation and burst. Their results indicated that $L / a$ in a two-dimensional extension differs by at most $2 \%$ from that predicted by the axisymmetric theory. The additional complexity in computing $L / a$ using the results of Hinch \& Acrivos (1979) was therefore judged by us to be unnecessary, and in comparisons between the experimental and predicted deformation curves ( $L / a$ vs. C), the results of the axisymmetric theory were used. The burst criterion for drops of low viscosity in two-dimensional extensional flow is nearly identical to the prediction of the axisymmetric theory:

$$
C_{\mathrm{c}} \lambda^{1}=0.145 \text {. }
$$

This result was used in comparisons to the experimental burst data for the $\alpha=1$ case shown in figure 25. 
Extension of the slender-drop theories to other two-dimensional flows $(0<\alpha<1)$ has not been attempted to date. The analysis is complicated by the fact that the cross-section of the drop is not only non-circular, but the position of the centreline is not known a priori. However, the analysis of Hinch \& Acrivos (1979) for twodimensional-straining flow suggests that as long as the cross-sectional area is comparable to that of a drop in axisymmetric extensional flow, the details of the cross-sectional shape will not be particularly important. Also, from the experimental observations, we know that long slender drops, for which this theory is expected to apply, align with the exit streamline of the flow field for $0<\alpha<1$. At this orientation they experience an 'effective strain rate' equal to $G \alpha^{\frac{1}{2}}$ (see $§ 5.3$ ). These facts suggest the possibility of an approximate ad hoc theory for intermediate flows based upon the rigorous theoretical results of Hinch \& Acrivos (1979) for $\alpha=1$. In this ad hoc approach, we assume that the features of the deformation and burst process for $\alpha=0.8,0.6,0.4$ and 0.2 are similar to those for $\alpha=1$, but with an effective strain rate $G \alpha^{\frac{1}{2}}$ replacing $G$. Thus an approximate deformation curve should be obtained from (34) with $G \alpha^{\frac{1}{2}}$ replacing $G$. 'The results of this calculation are compared to the experimental observations for $\lambda=0.001$ in figure 24. For predictions of the bursting point for intermediate flows, a similarly adapted version of (35) was employed:

$$
C_{\mathrm{c}} \lambda^{\frac{1}{6}}=\frac{0.145}{\alpha^{\frac{1}{2}}}
$$

The predictions from this equation are compared to the experimental data for $\alpha=0.8$, $0.6,0.4$ and 0.2 in figure 28 .

\section{Results and comparison with theory}

In the next two sections, we present the results of our experimental measurements. We begin in $\$ 5.1$ with a description of drop shapes at steady state. Rather than presenting all available data (which can be found in Bentley 1985) we initially concentrate on five cases representative of the behaviour for small, medium, and large values of the viscosity ratio. Following this, we present all available data from the present study for steady deformation and orientation as a function of capillary number with $\alpha=0.2,0.4,0.6,0.8$ and 1.0 , and $1.08 \times 10^{-3} \leqslant \lambda \leqslant 57$. These data illustrate that changes of the flow type, while holding the viscosity ratio constant, have little qualitative influence on the behaviour of the drop, at least for drops of moderate to low viscosity ratio, i.e. $\lambda \leqslant O(1)$. Finally, in $\$ 5.2$, we present data on the conditions for drop burst.

\subsection{Drop deformation}

For very low values of the capillary number $\mathbb{C}$ the drop deformation was similar for all viscosity ratios and flow types. All drops were deformed into ellipsoids aligned along the principal axis of extension (the $x$-axis of figure 2 ). As the shear rate was increased, however, the deformation characteristics became strongly dependent upon the viscosity ratio and the rotational character of the applied flow. We begin by considering the experimental results for low-viscosity-ratio drops $(\lambda<0.02)$.

\subsubsection{Low-viscosity-ratio drops}

Low-viscosity-ratio drops $(\lambda<0.02)$ required quite large values of the capillary number for burst, and were able to attain steady shapes which were highly deformed. The lower the viscosity ratio, the greater the sustainable steady deformation 
and the greater the capillary number at the point of burst. As the shear rate was increased beginning with initially small values, the radius of curvature at the ends of the drop decreased, and eventually the ends appeared to be pointed. The transition from ellipsoidal deformation to pointed ends occurred at $C(1+\alpha)$ approximately equal to 0.5. The ends were more cusp-like for lower-viscosity drops, and for all pointed drops the ends became sharper as the shear rate increased. The drop orientation angle monotonically approached the angle of the exit streamline of the flow field $\left(-3.2^{\circ},-7.2^{\circ},-12.7^{\circ}\right.$ and $-20.9^{\circ}$ for $\alpha=0.8,0.6,0.4$ and 0.2 , respectively) as the shear rate increased, and the drops became more elongated. Steady aspect ratios as high as 20 were observed for the lowest-viscosity-ratio systems studied. Even in the most rotational flow considered $(\alpha=0.2)$, the centreline of the drop remained straight, without any signs of the $S$ shape which is characteristic of low-viscosity-ratio drops in simple shear flows (Taylor 1934; Grace 1971; Torza et al. 1972).

At the point of burst, the drops no longer attained a steady shape, but extended continuously in time with the ends remaining pointed, becoming drawn into a thread of essentially constant radius. The 'tip-streaming' phenomenon reported by previous researchers (Taylor 1934; Rumscheidt \& Mason 1961; Torza et al. 1972; Grace 1971) was not observed in our experiments. It is possible that the magnification of our optical equipment was insufficient to resolve tiny drops being ejected from the drop ends. However, Torza et al. (1972) reported a correlation between tip streaming and the rate of change of the shear rate. They indicated that tip streaming occurred when the shear rate was changed rapidly, but apparently was suppressed for low $\mathrm{d} G / \mathrm{d} t$. Absence of tip streaming in our experiments (if it could be proven) would support this conclusion, since care was taken to increase the shear rate slowly to avoid transient phenomena.

Figure 5 shows a plot of the deformation parameter $D_{\mathrm{f}}$ and orientation angle $\theta$ versus capillary number for steady deformed drops in a flow with $\alpha=0.8$ and a viscosity ratio of $1.08 \times 10^{-3}$. The behaviour of this drop in flows with other values of $\alpha(0.2,0.4,0.6$ or 1.0$)$ is qualitatively identical to that illustrated here for $\alpha=0.8$. The error bars shown in the figure are typical of the experimental uncertainties in $D_{\mathrm{f}}$ and $\mathbb{C}$ in all of the deformation plots. The solid line shows the predictions of the $O(\epsilon)$ small-deformation theory of Barthès-Biesel \& Acrivos (1973a), and the dashed line represents their $O\left(\epsilon^{2}\right)$ theory. The asterisks terminating the theoretical lines indicate the theoretically predicted point of breakup at each level of approximation. In this case, it can be seen that both of the small-deformation theories agree with the observed deformation within the experimental error only for small values of capillary number, where $D_{\mathrm{p}}$ is essentially linear with $C$ as predicted by the classic analysis of Taylor (1934). The $O(\epsilon)$ and $O\left(\epsilon^{2}\right)$ corrections to the Taylor result predict a positive curvature in $D_{\mathrm{f}}$ vs. $C$, with the $O(\epsilon)$ theory predicting a larger deformation at a given $\mathbb{C}$ and a lower critical $\mathbb{C}$ at burst than the $O\left(\epsilon^{2}\right)$ theory. Although the $O\left(\epsilon^{2}\right)$ theory is in slightly better agreement with the experiment, the predicted burst point $\left(\mathbb{C}_{\mathrm{c}}=0.280\right)$ still differs from the observed bursting point $(0.51)$ by $45 \%$ ! The experimental deformation curve exhibits positive curvature at low $\mathbb{C}$, but has an inflexion point at about $C=0.3$, which is close to the value at which the transition to pointed ends occurs and where the $O\left(\epsilon^{2}\right)$ theory fails. The rather poor agreement between the predictions of the small-deformation theory and the experiment (except at low $\mathbb{C}$ ) is not surprising, since low-viscosity-ratio drops require high $\mathbb{C}$ for burst, and exhibit highly-deformed steady shapes, while the theory is valid only when $D_{\mathrm{f}}$ is small. We would expect a more accurate prediction for the elongated steady shapes from the slender-drop theory. 


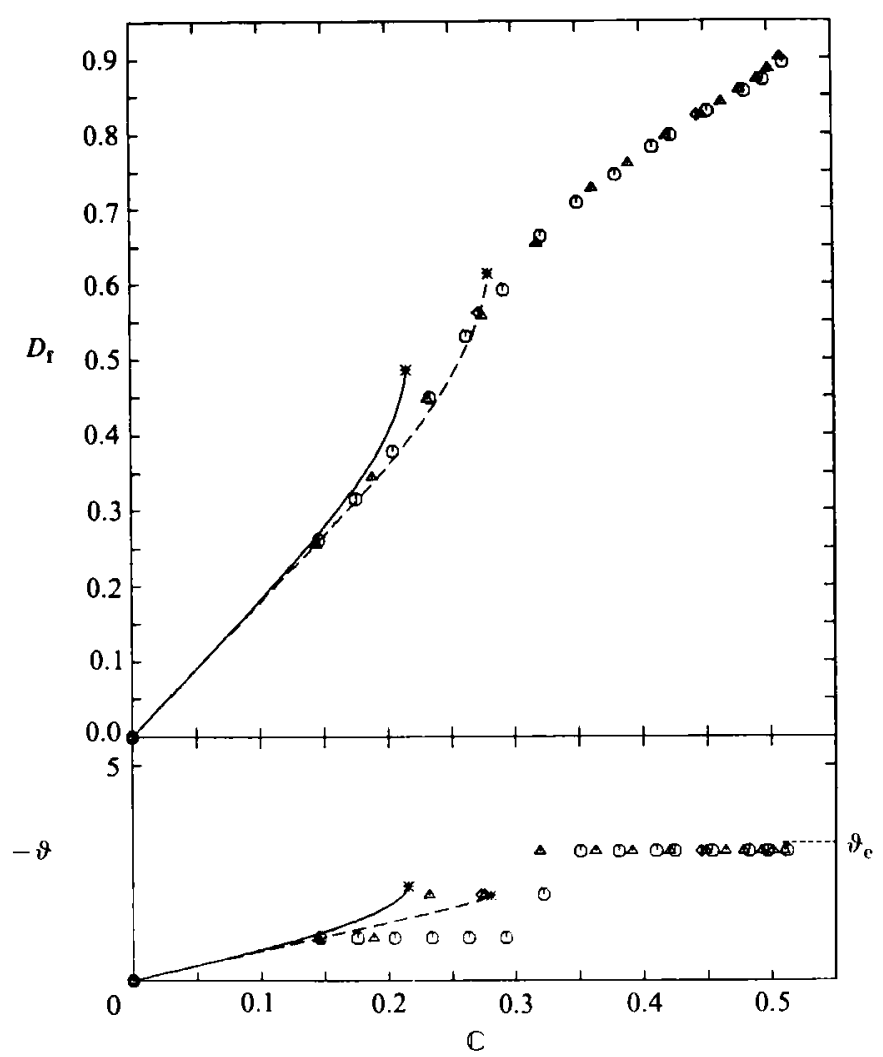

Figure 5. Deformation curve for $\alpha=0.8, \lambda=1.08 \times 10^{-3}$. Symbols, experiment; ,$- O(\epsilon)$ theory; ---, $O\left(\epsilon^{2}\right)$ theory.

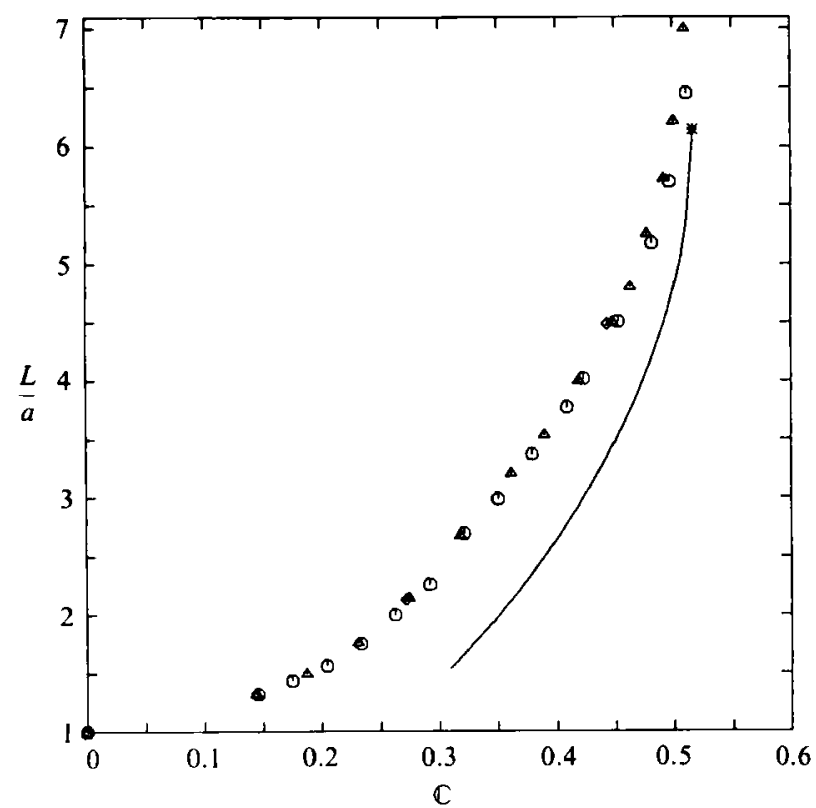

Figure 6. Dimensionless length $v s . \mathbb{C}$ for $\alpha=0.8, \lambda=1.08 \times 10^{-3}$. Symbols, experiment; - large-deformation theory. 


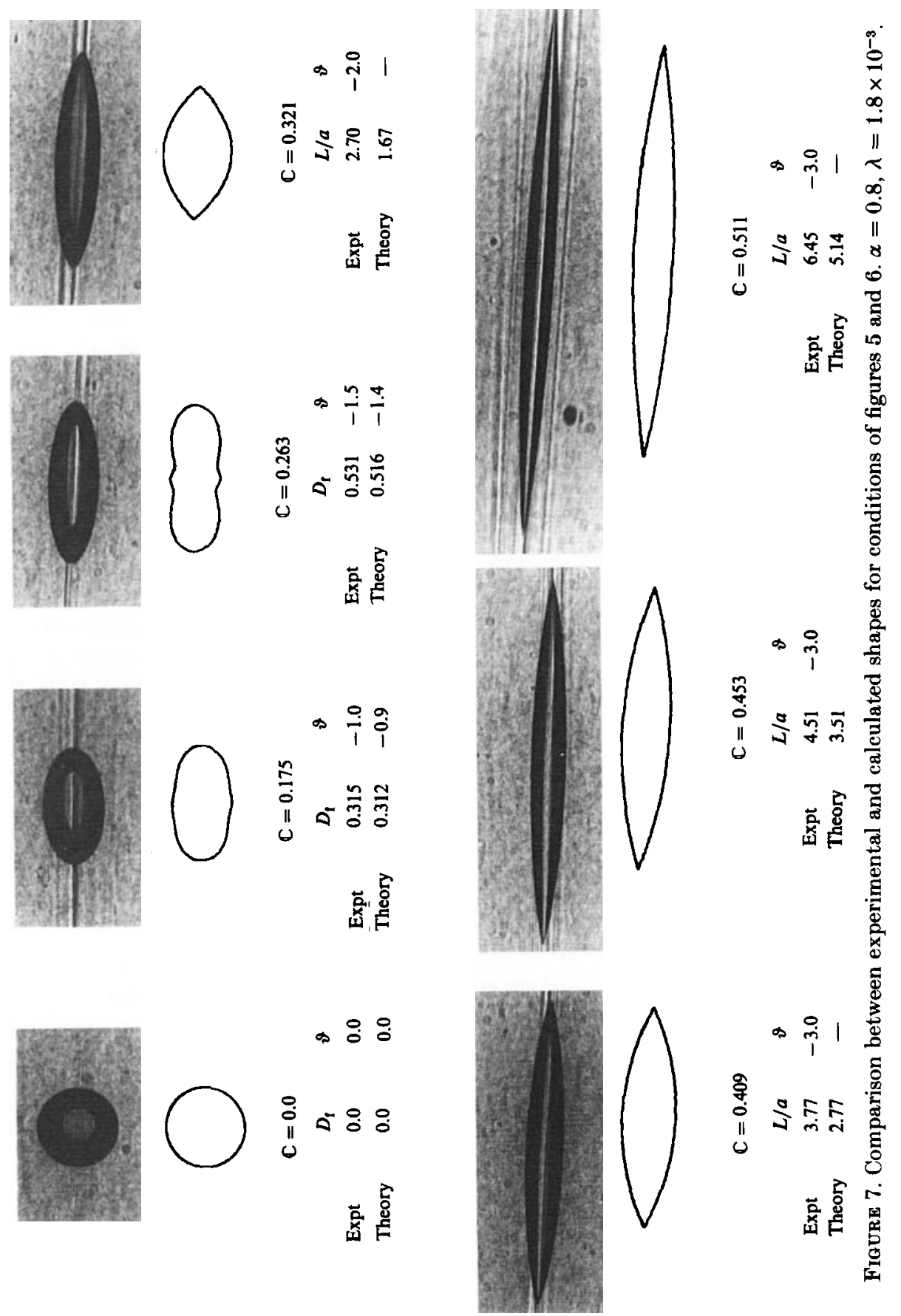


Figure 6 shows the dimensionless length $L / a$ plotted versus $\mathbb{C}$ for the same experimental conditions. The curve represents the predictions of the slender-drop theory. The agreement between the theory and the experiment improves with increasing deformation, as is expected since the theory is asymptotically valid as $L / a \rightarrow \infty$. The predicted bursting point, $\mathbb{C}_{\mathrm{c}}=0.513$, compares favourably with the observed value, 0.51 .

Figure 7 shows a comparison between actual photographs of the deformed drops and the predictions of the small-deformation and slender-drop theories. The lines visible in the photographs are caused by small refractive index gradients in the suspending fluid which arise from minute temperature gradients. They appear along the exit streamlines of the flow, where fluids from opposite sides of the tank meet. The second and third photographs are compared with the $O\left(\epsilon^{2}\right)$ small-deformation theory, and the last four with the slender-drop theory. The comparison with the $O\left(\epsilon^{2}\right)$ theory is reasonable for the second photograph, with $C=0.175$ and $D_{\mathrm{f}}=0.31$, although there are larger differences between the experimental and calculated shapes than is suggested by the $1 \%$ difference between the experimental and predicted values of $D_{\mathrm{p}}$ for this case. The ends of the calculated shape are more blunt, and the approximations inherent in representing the shape with only tensors of order two and four are apparent from the slight 'bump' in the sides of the drop. As the capillary number is increased, the shapes predicted by the small-deformation theory become increasingly unrealistic, as can be seen from the third photograph, at $C=0.265$, where the predicted shape bears only a superficial resemblance to that found experimentally (the length and breadth, and hence $D_{\mathrm{f}}$ are comparable). The $O\left(\epsilon^{2}\right)$ small-deformation theory predicts burst at $C_{c}=0.28$, so for the remainder of the photographs the comparison is to the predictions of the slender-drop theory. Qualitatively, the predicted and observed shapes are similar, especially for $C \geqslant 0.409$. For the most extended drop in this sequence, the difference between the theoretical and experimental $L / a$ was about $20 \%$. The predictions of the slender-drop theory would undoubtedly improve for lower-viscosity-ratio systems, or for this system at larger values of $L / a$.

\subsubsection{Intermediate-viscosity-ratio drops}

All fluid systems with the viscosity ratio between 0.02 and 2.0 behaved in a qualitatively similar manner. The ends of the drops remained rounded to the point of burst (with less viscous drops exhibiting a lower radius of curvature), and the orientation angle varied monotonically from zero (aligned with the $x$-axis) at low capillary number to alignment with the exit streamline of the flow as the bursting point was approached. The critical capillary number and the maximum stable deformation both decreased as the viscosity ratio increased for all flow types investigated. As the critical shear rate was approached, drop burst was indicated by the appearance of 'flat sides', where the radius of curvature in the $x-y$ cross-section became infinite along the length of the drop. Once this point was reached, no steady shapes seemed possible. The drop sides became concave and the drop was pulled into a thread, with the smallest radius at the central portion. The shapes of drops undergoing this transient motion at the critical capillary number were surprisingly similar over a wide range of viscosity ratios (all $\lambda>0.02$ ). The qualitative aspects of the burst phenomena and the maximum stable deformation seemed independent of the flow type. The mode of burst observed corresponds roughly to the 'B-2' mode described by Rumscheidt \& Mason (1961) and Torza et al. (1972). This is consistent 


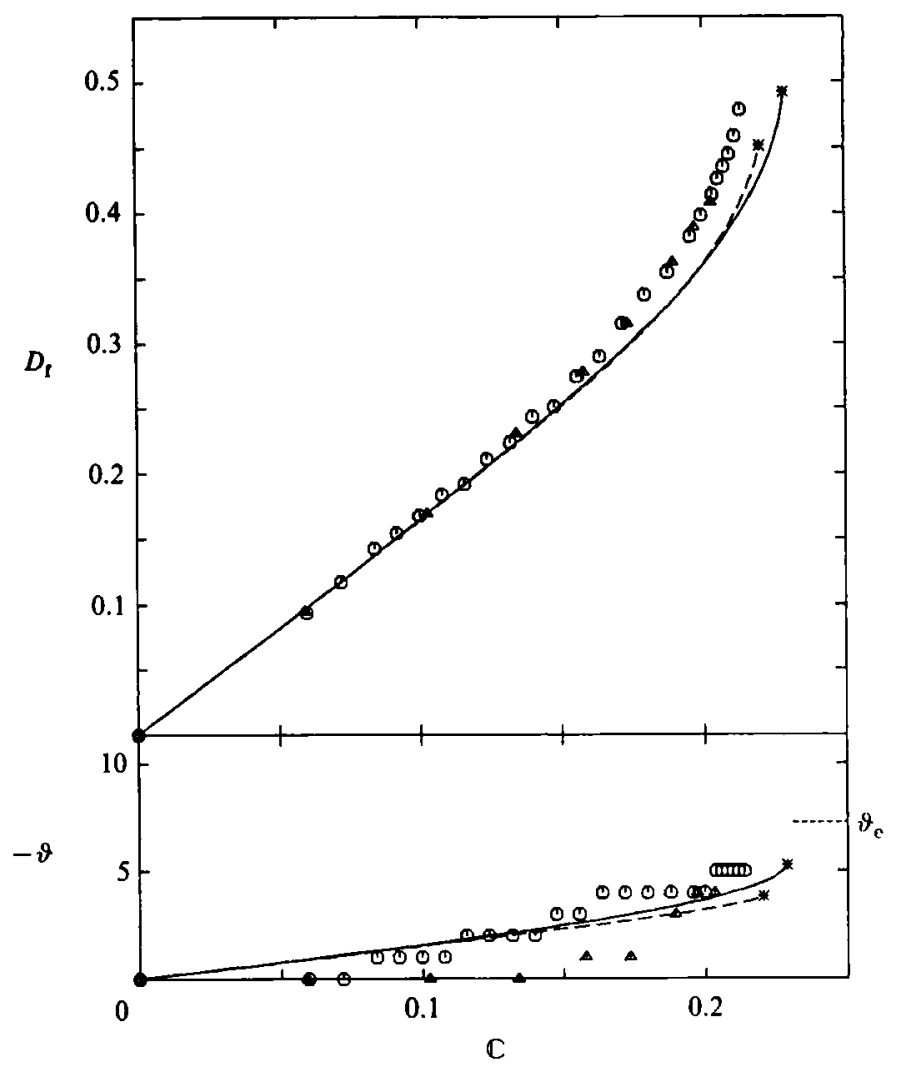

Figure 8. Deformation curve for $\alpha=0.6, \lambda=0.118$. Symbols, experiment;,$- O(\epsilon)$ theory; ,$-- O\left(\epsilon^{2}\right)$ theory.

with the descriptions of burst reported by Taylor (1934), Rumscheidt \& Mason (1961), and Grace (1971) for drops in two-dimensional irrotational flow.

Figure 8 shows a plot of $D_{\mathrm{f}}$ and $\theta$ versus $\mathbb{C}$ for $\lambda=0.118$ in a flow with $\alpha=0.6$. In this case, the predicted values of the scalar deformation measure, $D_{\mathrm{f}}$, are in surprisingly good agreement with the experimental observations, even up to $D_{\mathrm{p}}=0.5$. The predictions of the $O(\epsilon)$ and $O\left(\epsilon^{2}\right)$ theories are similar, with the $O\left(\epsilon^{2}\right)$ theory predicting slightly higher deformation near the bursting point, and slightly lower $\mathbb{C}_{\mathrm{c}}$ at burst. The data lie above both calculated deformation curves, with burst occurring at $\mathbb{C}_{\mathrm{c}}=0.21$, compared to 0.228 and 0.220 predicted by the $O(\epsilon)$ and $O\left(\epsilon^{2}\right)$ theories, respectively. The orientation angles predicted by the theory agree reasonably well with those observed, particularly in view of the difficulty in accurately measuring these angles from the photographs of the drops.

Figure 9 shows the comparison between the actual drop shapes and those predicted by the $O\left(\epsilon^{2}\right)$ small-deformation theory for the same parameters as figure 8 . The agreement is satisfactory for $\mathbb{C}$ up to about 0.2 , at which point the drops assume shapes which could only be satisfactorily represented by including higher-order deformation tensors. The 'bumps' in the theoretically calculated drop shapes are present in this case also (and, as pointed out by Barthès-Biesel \& Acrivos (1973a), for all but high viscosity ratios), but are less pronounced than for lower viscosity ratios. In the final photograph in the sequence, the drop is shown bursting, while the theory predicts a steady shape for that value of $\mathbb{C}$. 

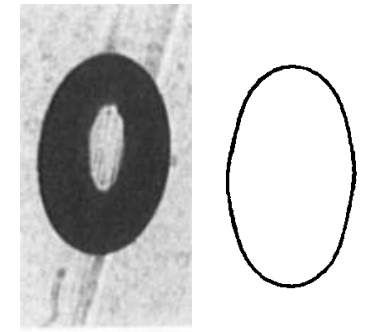

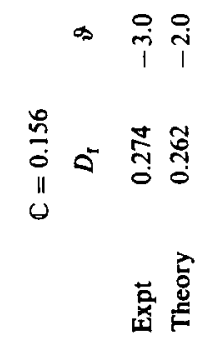

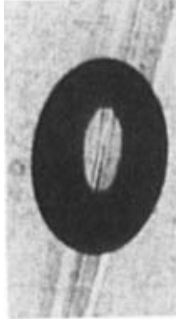

$$
\text { a } \stackrel{0}{i} \frac{0}{1}
$$

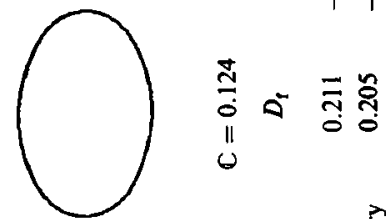

蒙总
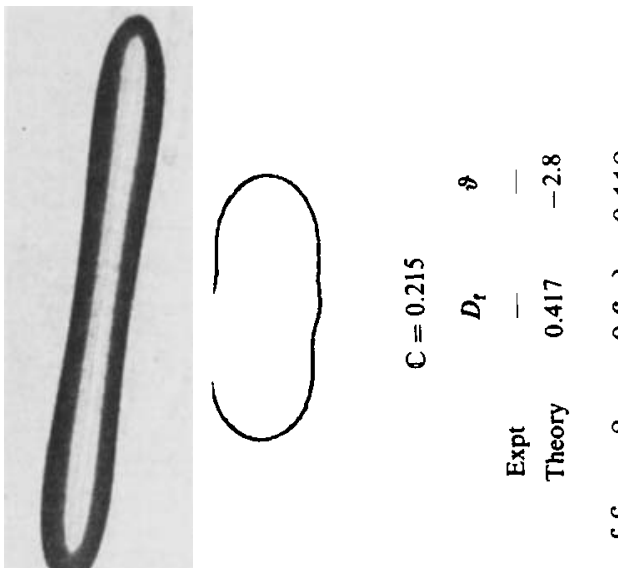

$\rightarrow \stackrel{\circ}{i} \stackrel{\infty}{1}$
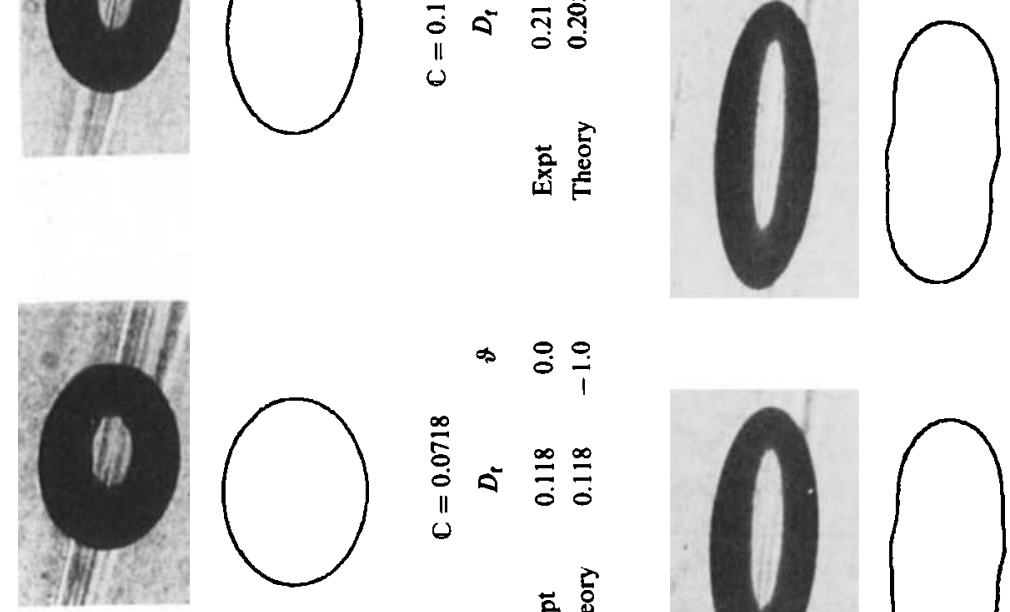

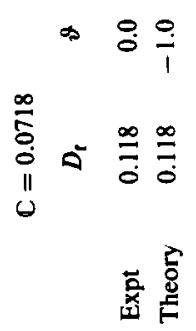

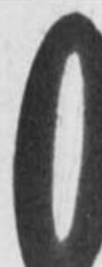

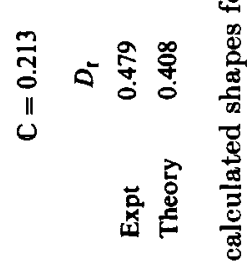
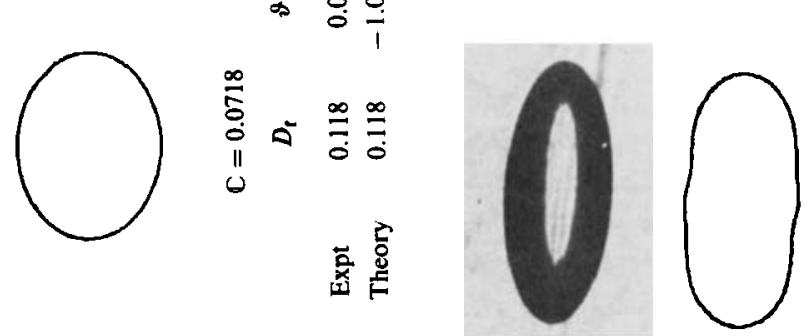

ญ̊ำ

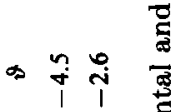

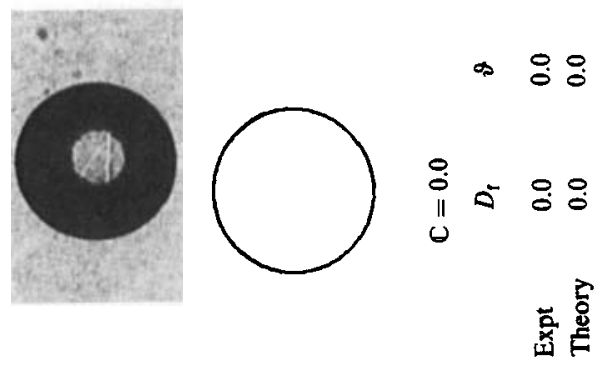

蕺趈
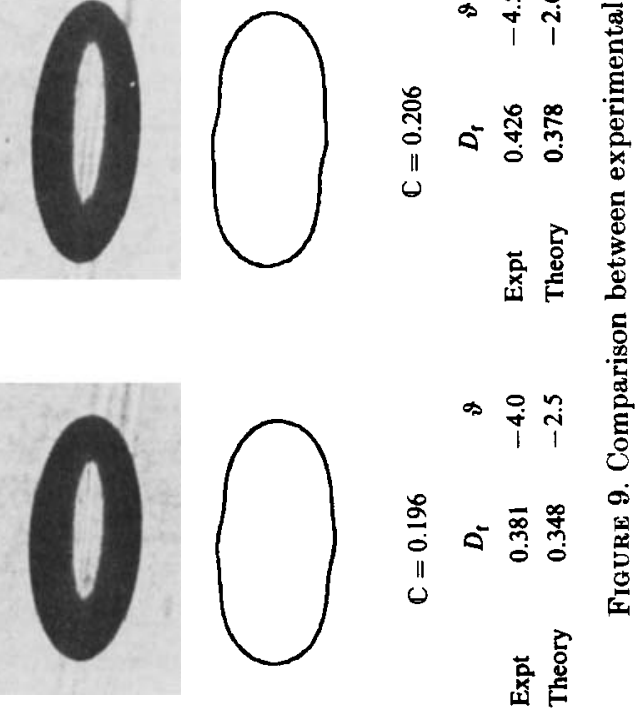


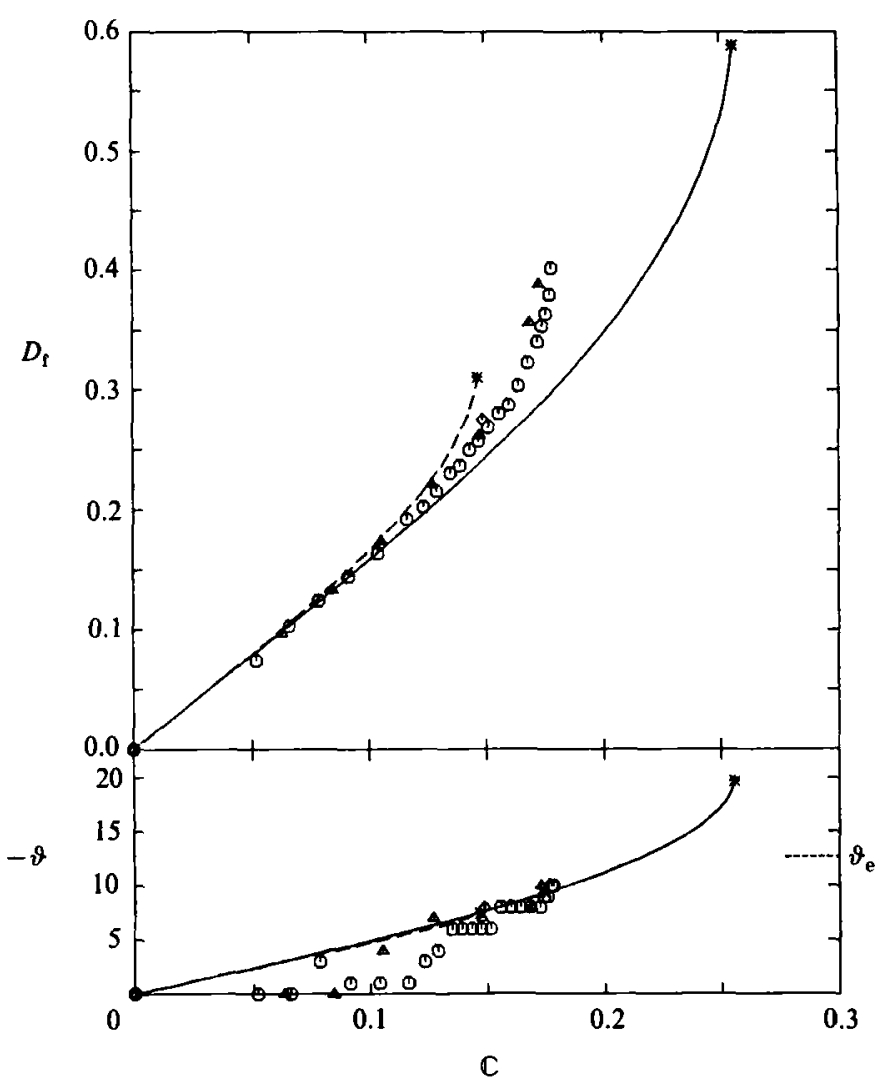

Figure 10. Deformation curve for $\alpha=0.4, \lambda=1.58$. Symbols, experiment; - $O(\epsilon)$ theory; ,$-- O\left(\epsilon^{2}\right)$ theory.

Experimental observations for a second 'intermediate-viscosity-ratio' case, $\lambda=1.58, \alpha=0.4$, are compared to the calculated deformation and orientation in figure 10 . In this case, at a given $C$, the $O\left(\epsilon^{2}\right)$ small-deformation theory predicts a deformation which is greater than that observed, while the $O(\epsilon)$ theory predicts lower-than-observed deformations. Thus the experimental points are 'bracketed' by the two theoretical curves, with neither supplying a particularly accurate prediction. The bursting point $\left(C_{c}=0.178\right)$ is underestimated by $18 \%$ by the $O\left(\epsilon^{2}\right)$ theory and overestimated by $43 \%$ by the $O(\epsilon)$ theory. This bracketing of the data, with an upper bound on the bursting point supplied by the $O(\epsilon)$ result and a lower bound supplied by the $O\left(\epsilon^{2}\right)$ result, is typical of the small deformation theory for $0.5<\lambda<3$.

Photographs for this case are compared to shapes calculated from the $O\left(\epsilon^{2}\right)$ theory in figure 11. The theory predicts drop burst at $C_{c}=0.146$, so no calculated shapes are included for the last three photographs in the sequence. The final photograph shows the drop bursting. The calculated shapes are in qualitatively good agreement with the experiment, as are the predicted orientation angles. From comparisons between the photographs of figures 7,9 , and 11, it is apparent that the ends of the deformed drops become blunter with increasing viscosity ratio, and that these shapes are more readily represented by the theory, which retains only second- and fourth-order deformation tensors. 


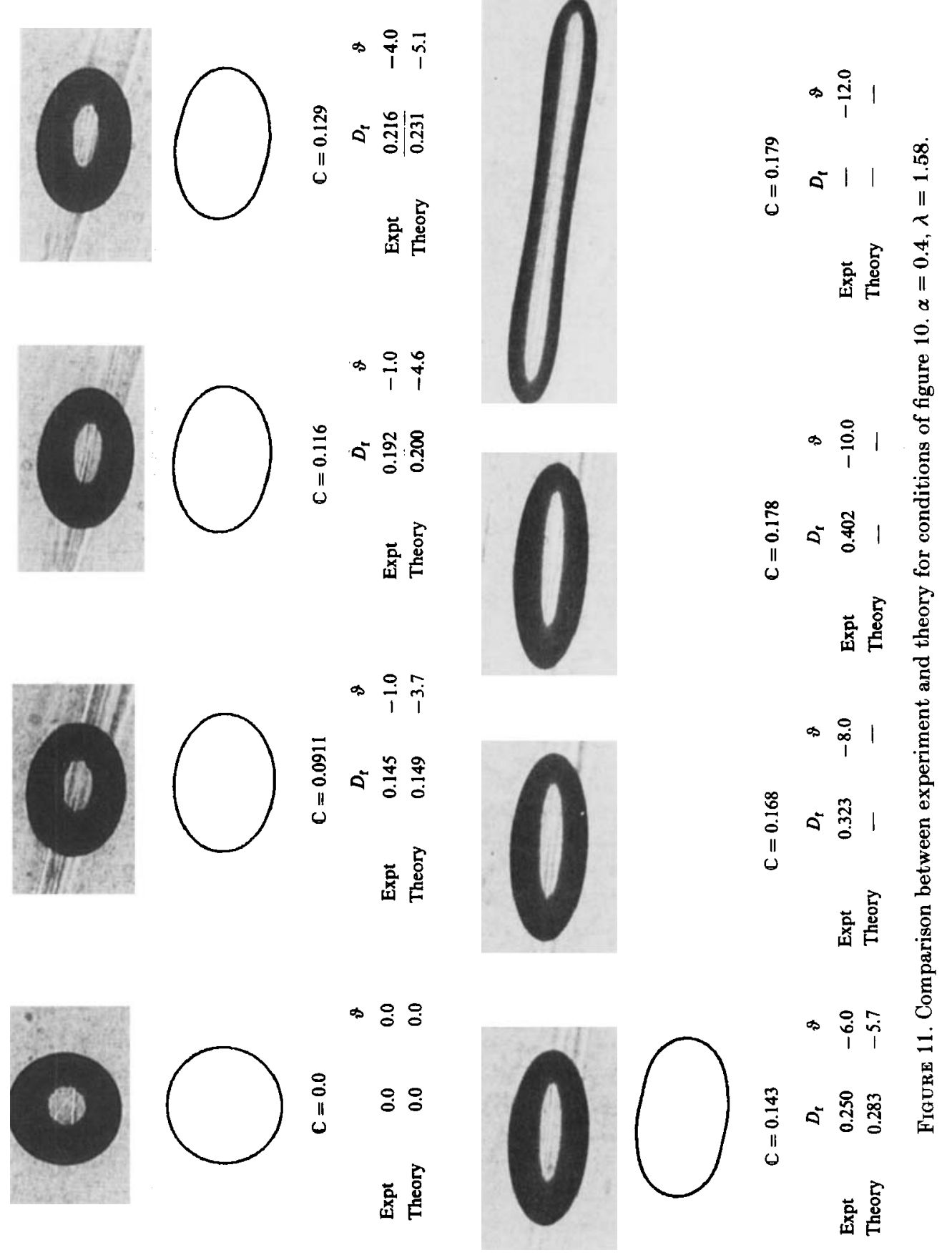




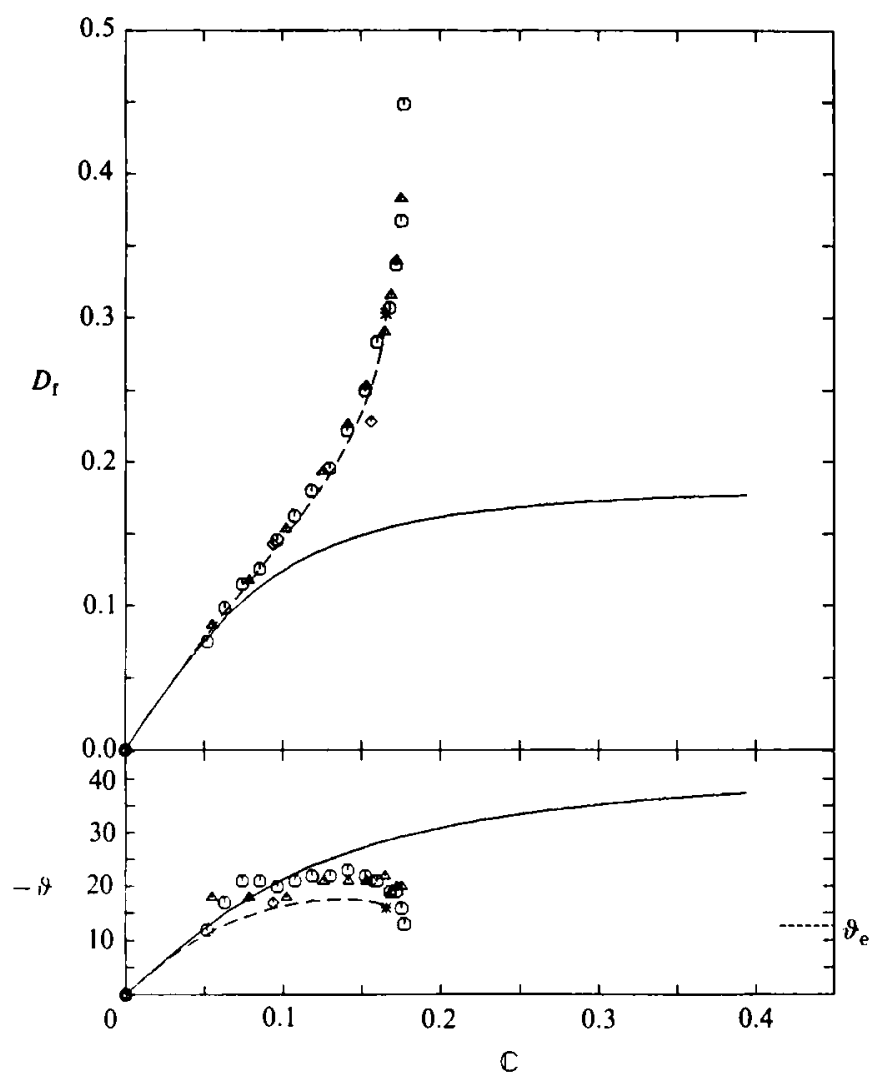

Figure 12. Deformation curve for $\alpha=0.4, \lambda=14.4$. Symbols, experiment;,$- O(\epsilon)$ theory; ,$-- O\left(\epsilon^{2}\right)$ theory.

\subsubsection{High-viscosity-ratio drops}

For drops of viscosity ratio greater than 3 , the vorticity in the flow field had pronounced effects on the drop orientation and deformation characteristics. For flows with vorticity, $(\alpha \neq 1.0)$, the drops were aligned with the $x$-axis only for very small deformations, with the magnitude of the orientation angle increasing rapidly with increasing shear rate. Indeed, at moderate deformations, the magnitude of the orientation angle even exceeded that of the exit streamline. Upon further increase of the shear rate, the magnitude of the orientation angle went through a maximum, and decreased to the angle of the exit streamline as the bursting point was approached. This behaviour was particularly evident for the more rotational flows considered, $\alpha=0.4$ and 0.2 . In those flows, limiting viscosity ratios were found, above which breakup was not possible. The limiting viscosity ratio for $\alpha=0.2$ was approximately 27, and for $\alpha=0.4$ the value was about 57 . The relationship between viscosity ratio, drop orientation, and bursting point is discussed in $\S 5.3$.

Figure 12 shows the deformation and orientation curves for a high-viscosity-ratio case, $\lambda=14.4$, in a flow with $\alpha=0.4$. In calculating $D_{\mathrm{f}}$ the fourth-order tensor terms have been discarded as discussed in $\$ 4.1$ above. In this case, the $O(\epsilon)$ theory proved virtually useless, predicting that the drop attains a steady shape without bursting as the shear rate increases. In contrast, the $O\left(\epsilon^{2}\right)$ theory predicts the deformation curve with surprising accuracy, and the predicted bursting point, $C_{\mathrm{c}}=0.165$, agrees 

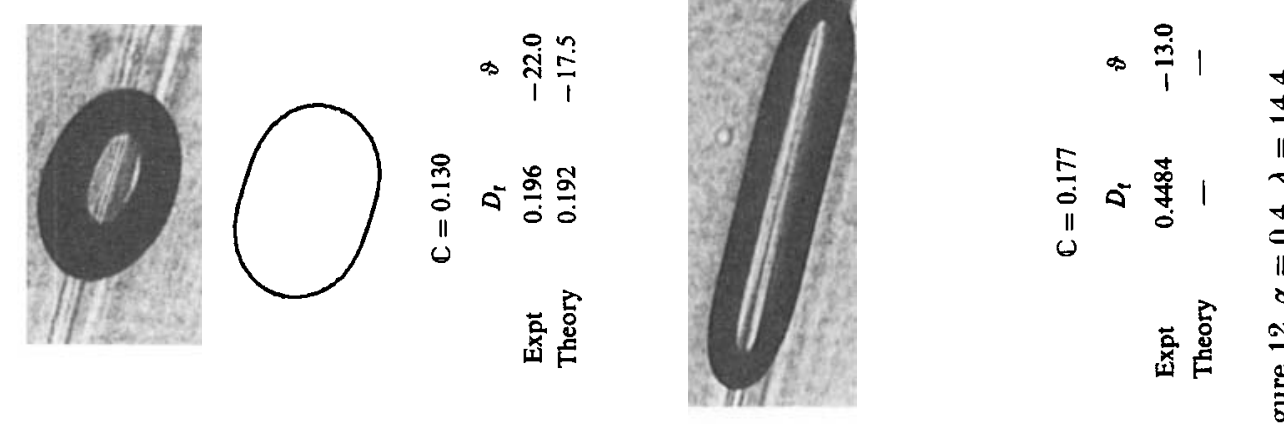

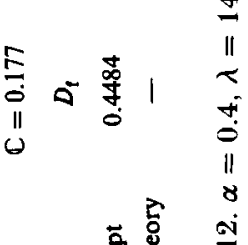

离总

焉

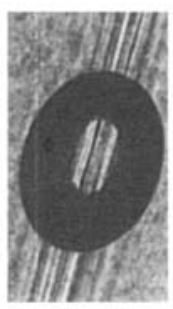

$\infty \stackrel{0}{0} \frac{\overrightarrow{0}}{1}$

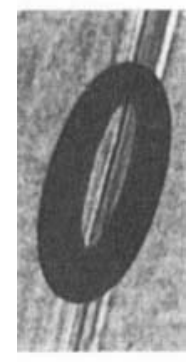

$\infty \stackrel{0}{\circ}$

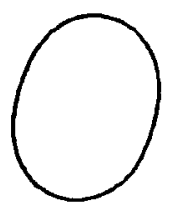

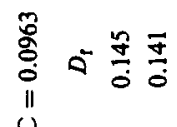

莕密

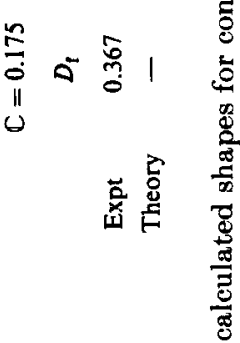

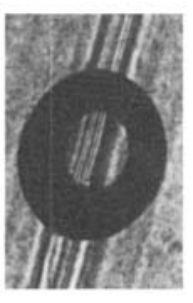

$\infty \stackrel{\sim}{=} \underset{1}{=}$

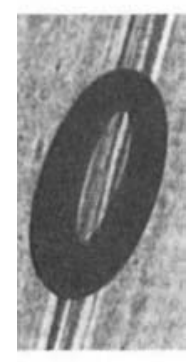

$\infty \frac{0}{1}$

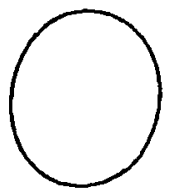

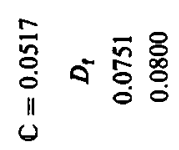

营总

点

营总

ఖ

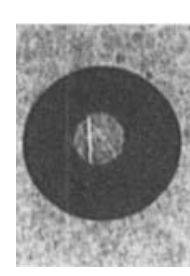

क 용요
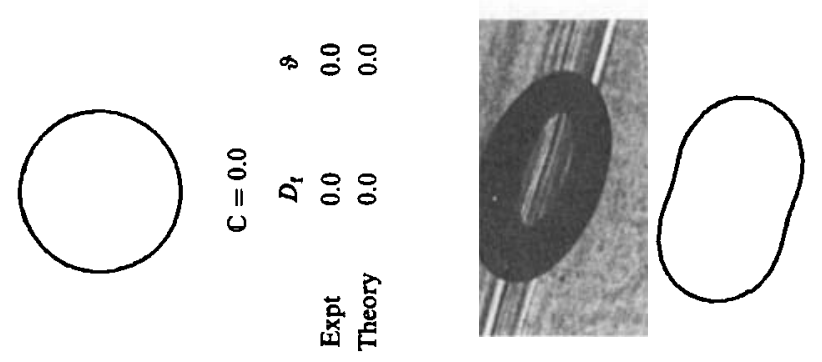

क 웅

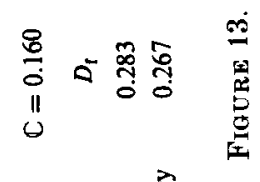

葛总 


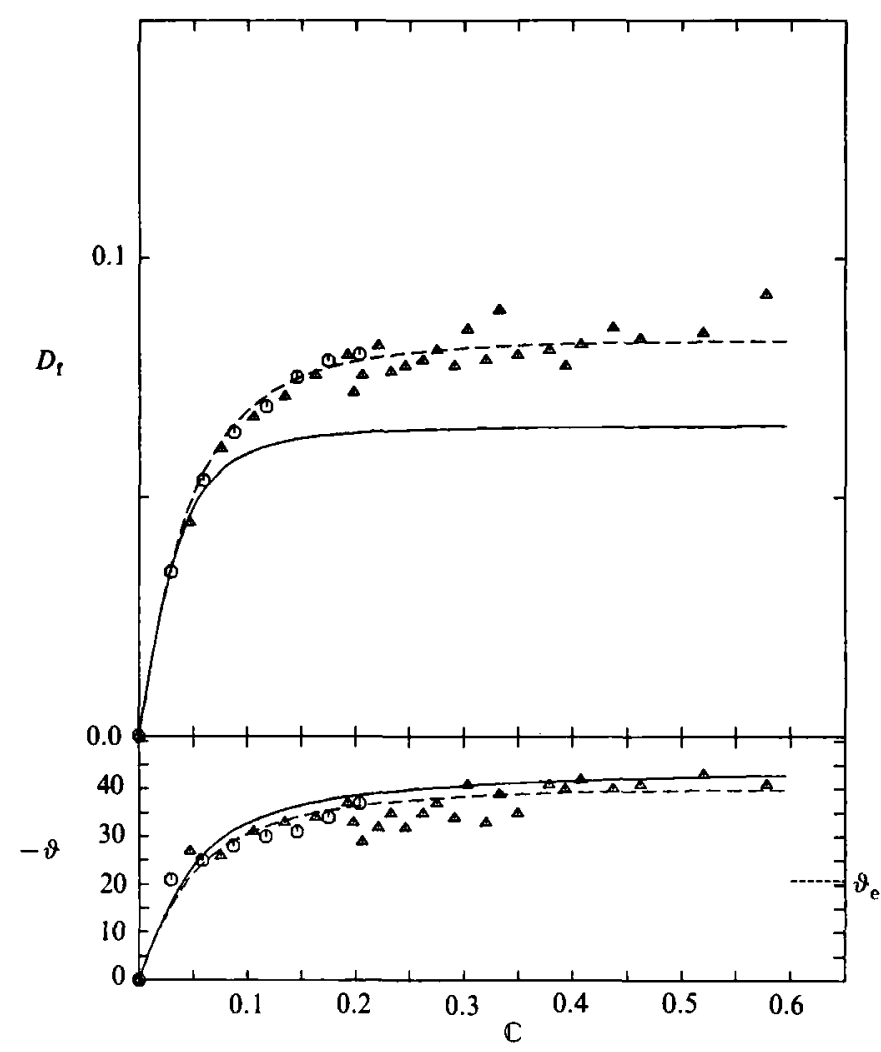

Figure 14. Deformation curve for $\alpha=0.2, \lambda=27.3$. Symbols, experiment;,$- O(\epsilon)$ theory; ,$-- O\left(\epsilon^{2}\right)$ theory.

well with the observed value of 0.176 . The critical deformation at breakup, however, is underestimated by the theory.

Both the experimental results and the predictions of the $O\left(\epsilon^{2}\right)$ small-deformation theory show the orientation angle $\theta$ going through a maximum with increasing shear rate as discussed in $\S 5.3$. The observed maximum magnitude of $\theta$ was $22^{\circ}$, in reasonable agreement with the theoretical prediction of $18^{\circ}$.

Figure 13 shows the comparison between the observed drop shapes and those calculated from the $O\left(\epsilon^{2}\right)$ theory for this case, $\lambda=14.4$. The comparison is good for $\mathbb{C}$ less than about 0.10 . The more eccentric shapes at higher $\mathbb{C}$ cannot be accurately represented by retaining only the second-order deformation tensor, but the calculation of the fourth-order tensor fails at high viscosity ratio as discussed in \$4.1. An accurate calculation of the fourth-order tensor would probably have improved the comparison for $\mathbb{C}=0.13$ and 0.16 , particularly in the latter case where the theory with only the second-order tensor predicts a steady drop shape with physically unrealistic concave sides.

Figure 14 shows the deformation and orientation curves for a case where no drop burst was observed, with a flow type of 0.2 and a viscosity ratio of 27.3. The predictions of the $O\left(\epsilon^{2}\right)$ theory (without the fourth-order tensor) were quite accurate, predicting a limiting deformation of about $\mathbf{0 . 0 8 3}$, compared to the observed $0.085 \pm 0.01$. The $O(\epsilon)$ theory predicted a limiting value of about 0.07 . The predicted limiting orientation angle for the $O\left(\epsilon^{2}\right)$ theory was about $-40^{\circ}$, in good agreement 


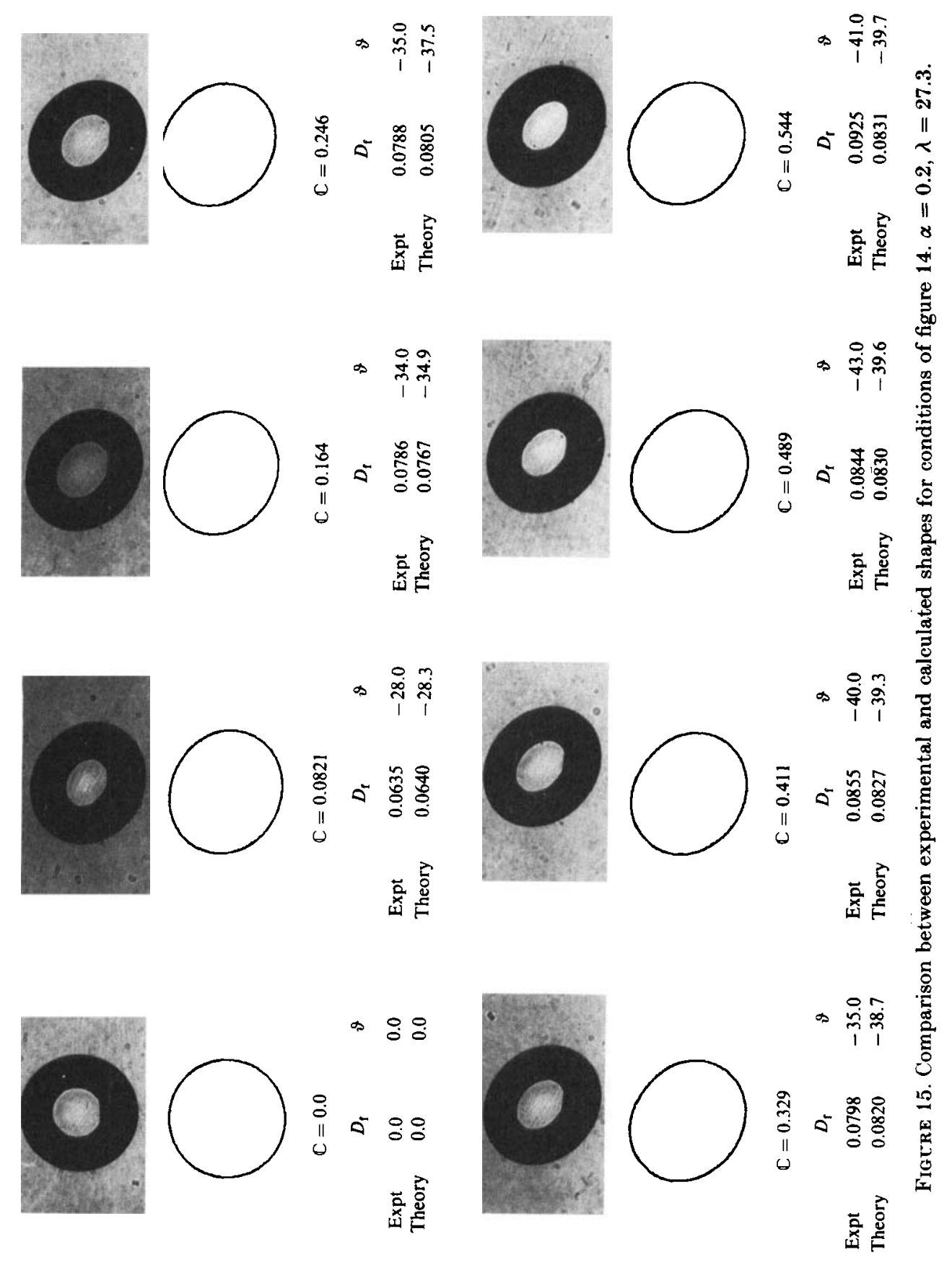




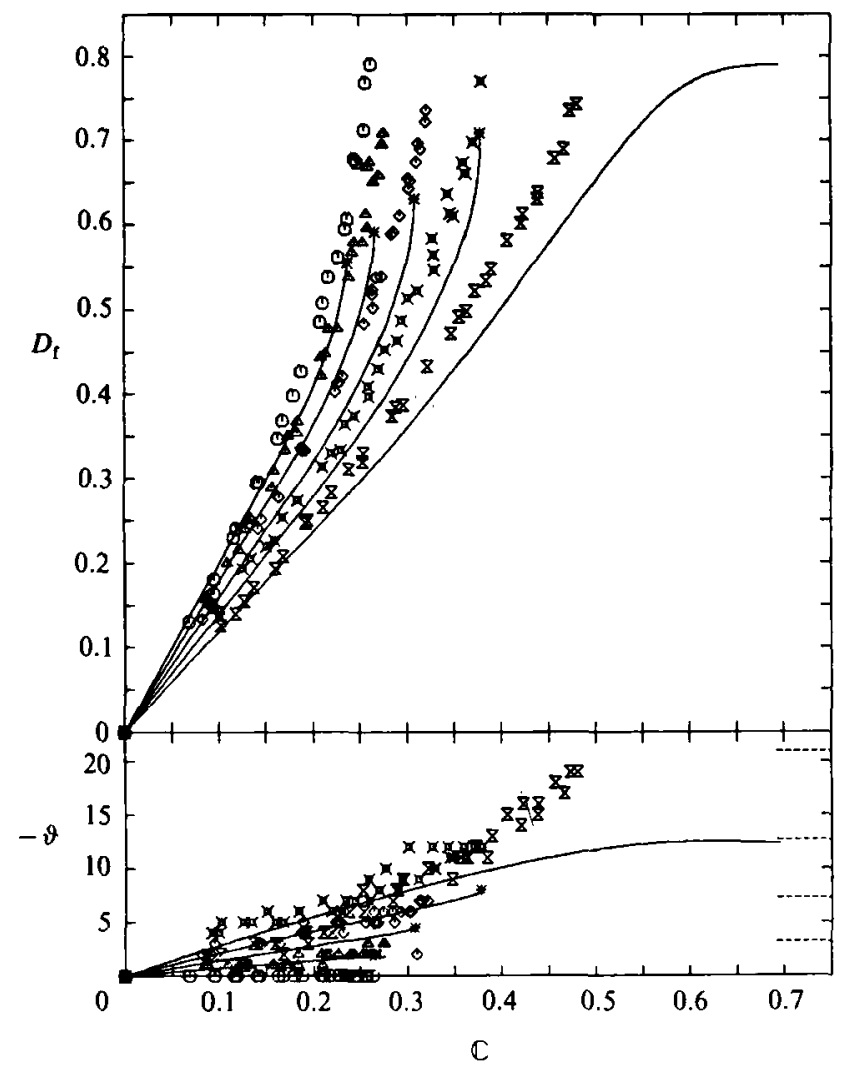

FiqURE 16. Drop deformation curves for different $\alpha$ for $\lambda=1.1 \times 10^{-2}: \mathbb{0}, \alpha=1.0 ; \triangle, \alpha=0.8$; $\diamond, \alpha=0.6 ; \not, \alpha=0.4 ; \mathrm{z}, \alpha=0.2 ;-, O\left(\epsilon^{2}\right)$ theory.

with the observations. At this orientation, the effective straining motion is apparently so low that the drop shape remains essentially constant. This is discussed in $§ 5.3$ below.

Finally, figure 15 shows the comparison in this dase between the photographs and the shapes predicted by the $O\left(\epsilon^{2}\right)$ theory. It can be seen that the deformation is small for all $C$, and thus the theoretical and experimental shapes are very similar.

\subsubsection{Flow-type effects}

Figures 16 to 23 are provided to show systematically the effect of flow type on drop deformation. Each figure shows experimentally measured values for $D_{\mathrm{f}}$ and the predictions of the $O\left(\epsilon^{2}\right)$ small-deformation theory at a particular viscosity ratio for five different flow types $(\alpha=1.0,0.8,0.6,0.4$ and 0.2$)$. The experimental points for different flow types are distinguished by different symbols, and in every case the theoretical curves go in order of decreasing $\alpha$ from left to right.

For low and intermediate viscosity ratios (figures 16-19), the qualitative behaviour of the drops is not highly dependent on the flow type. Deformation is smaller for lower $\alpha$ at fixed $\mathbb{C}$ mainly because the magnitude of the rate-of-strain tensor $(=G(1+\alpha))$ decreases with decreasing $\alpha$. Except for the lowest viscosity ratios $\left(\lambda=1.1 \times 10^{-3}\right.$ and $1.1 \times 10^{-2}$, where the $O\left(\epsilon^{2}\right)$ small-deformation theory prediets no drop burst for $\alpha=0.2)$ the $O\left(\epsilon^{2}\right)$ theory reflects the observed trends. For viscosity ratios above 2.80, the vorticity starts to have more dramatic effects, with the ratio of eapillary number 
268

B. J. Bentley and L. G. Leal
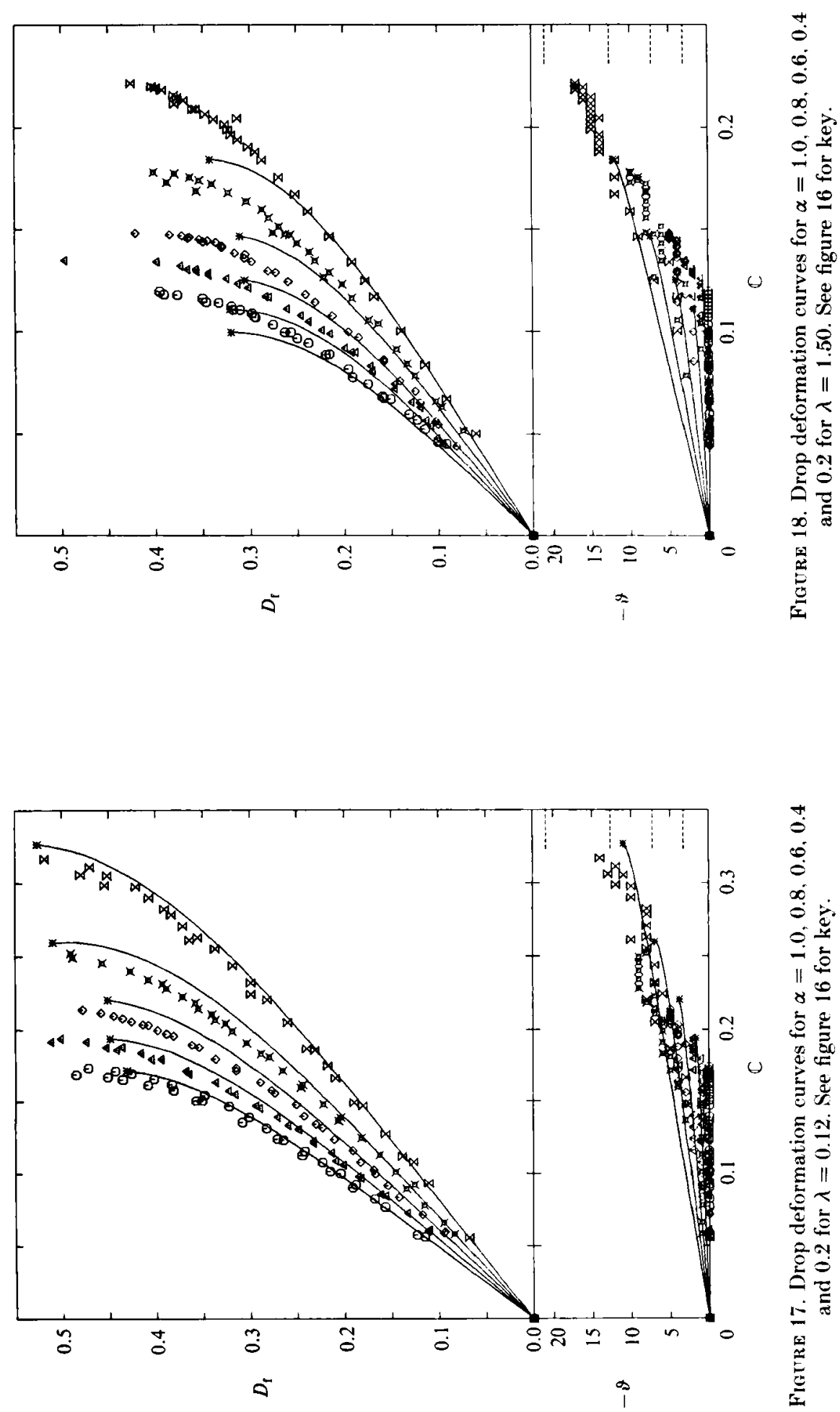

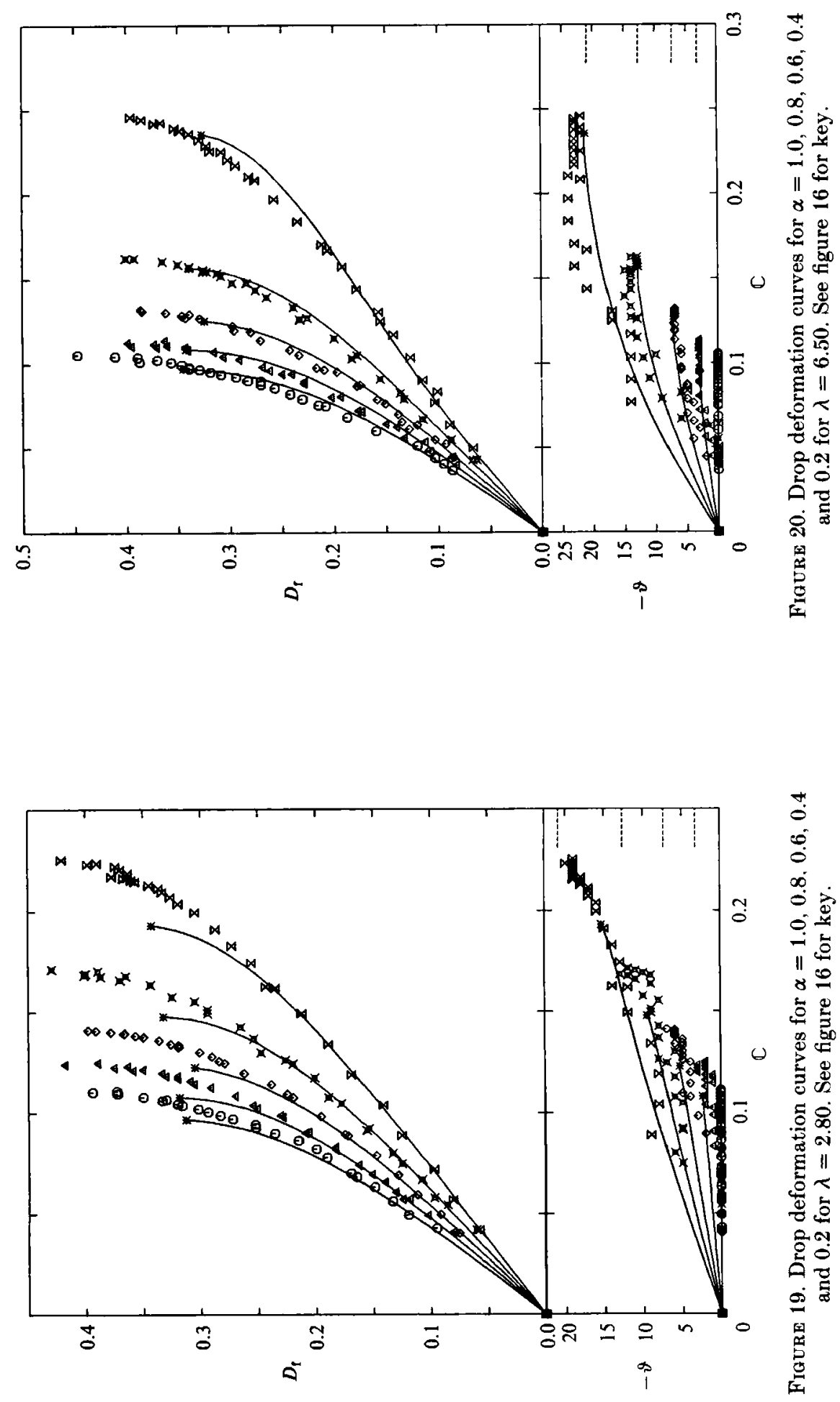

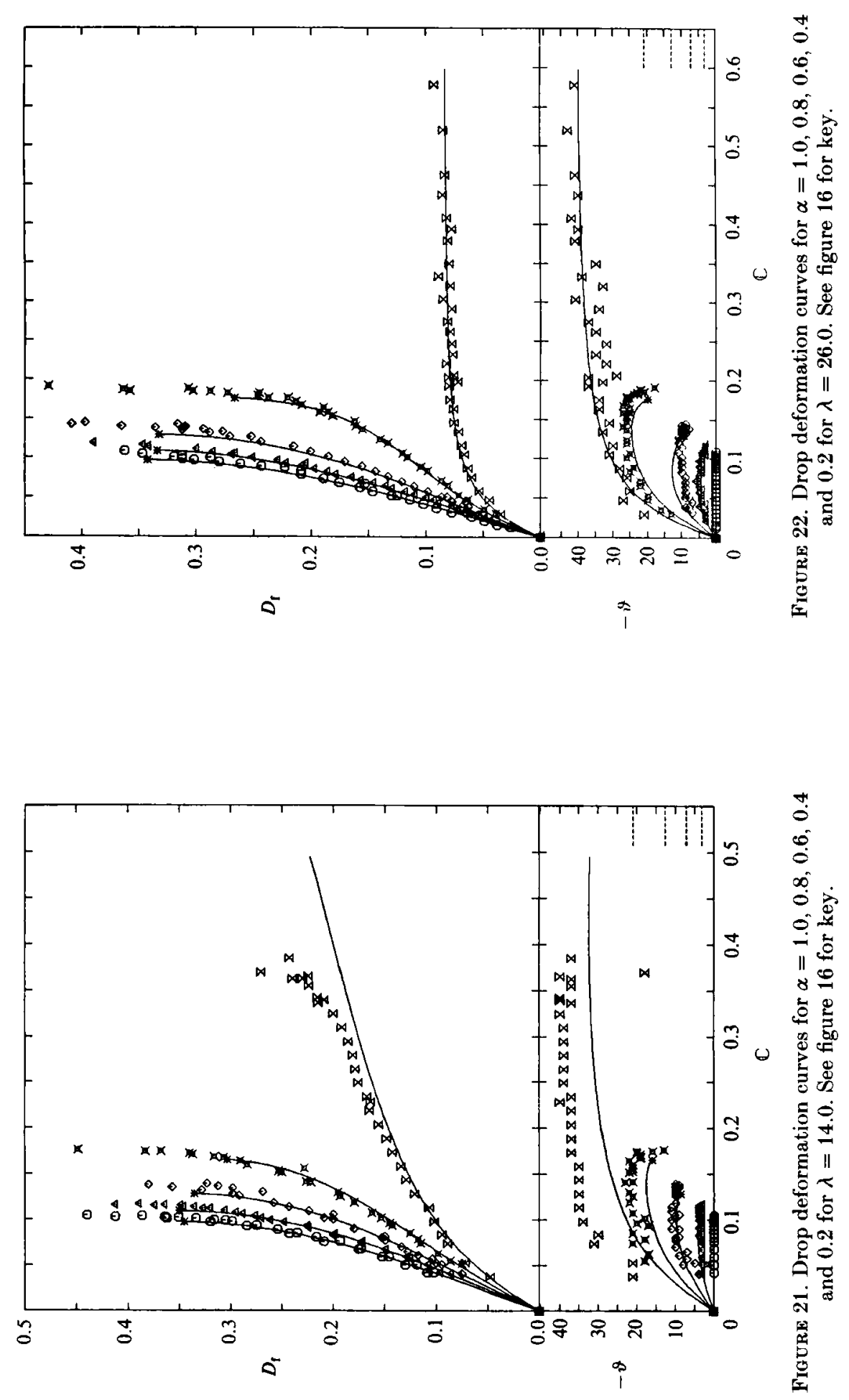


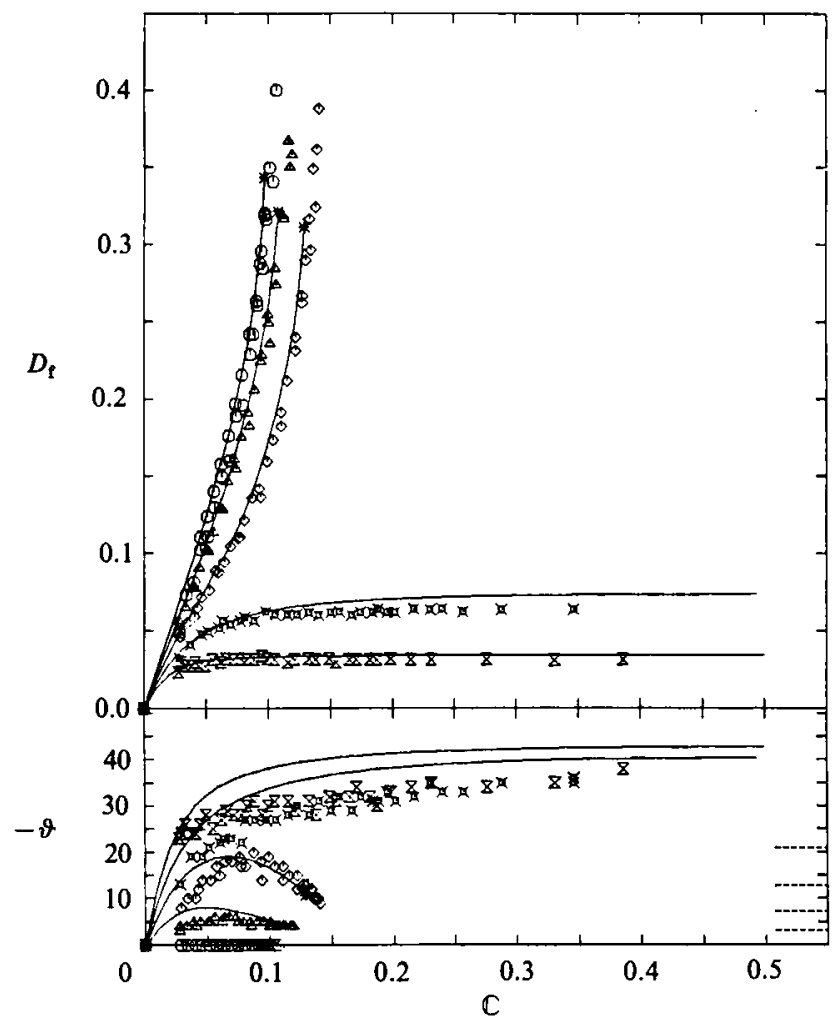

Figure 23. Drop deformation curves for $\alpha=1.0,0.8,0.6,0.4$ and 0.2 for $\lambda=57.0$. See figure 16 for key.

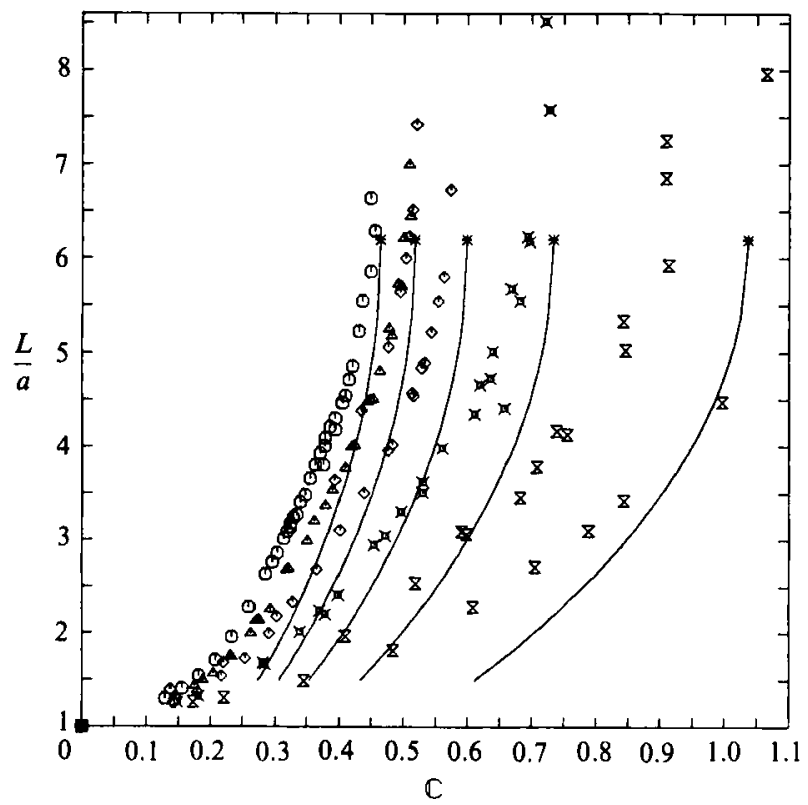

Figure 24. $L / a$ vs. $C$ for $\alpha=1.0,0.8,0.6,0.4$ and 0.2 for $\lambda=1.1 \times 10^{-3}$. Comparison with large-deformation theory, $0, \alpha=1.0 ; \Delta, \alpha=0.8 ; \diamond, \alpha=0.6 ; \varangle, \alpha=0.4 ; 8, \alpha=0.2 ;-$, large-deformation theory. 
required for burst in the rotational flows relative to that for $\alpha=1.0$ increasing, particularly for $\alpha=0.4$ and 0.2 . The results for drop burst as a function of viscosity ratio and flow type are discussed in detail in \$5.2.

Figure 24 shows the experimental results for each flow type for the lowest viscosity-ratio system $\left(\lambda=1.1 \times 10^{-3}\right)$, plotted as $L / a$ versus $C$ for convenient comparison to the large-deformation theory. The plot includes data for each flow type considered, as well as the predictions of the large-deformation theory described in $\$ 4.2$. As can be seen, the theory gives acceptable results near the burst point $(L / a$ about 7). Burst for the next-lowest viscosity-ratio system considered $\left(\lambda=1.1 \times 10^{-2}\right)$ occurred at $L / a$ of about 3.5 , and agreement with the slender-drop theory was poor, in keeping with the nature of the theory, which is valid only in an asymptotic sense for large $L / a$.

\subsection{Drop burst}

In this section we consider the conditions which lead to drop burst for a given fluid system. In fact, drop burst depends not only on the instantaneous flow conditions (i.e. flow type and capillary number), but on the entire time history of velocity gradient experienced by the drop. That is, drop burst is a functional over the history of $\nabla u$. For example, Torza et al. (1972) reported that drops could be made to burst in simple shear by a sudden increase in shear rate to a value below that required for burst when the shear rate was slowly increased. The same behaviour was observed in the theoretical study of Hinch \& Acrivos (1980).

A complete investigation of the drop-burst functional would be impossible, due to the many degrees of freedom available in specifying the velocity-gradient history. Thus, in this work, we limit our attention to one such history. Specifically, we consider only the case where the form of the velocity-gradient tensor is constant (fixed flow type) and the flow strength is increased very slowly, so that the drop goes through a progression of equilibrium states. The bursting point is defined as the shear rate for which no steady shape exists (if there is such a shear rate). This particular choice of flow history provides a sufficient condition for drop burst, in that any other approach to a constant flow field (e.g. a step increase in shear or a ramped shear rate) will result in drop burst at the same or lower final shear rate. Thus our results can be used to predict an upper bound for the size of a drop which can exist indefinitely in a given flow field.

Figure 25 shows the critical capillary number for burst $\mathbb{C}_{c}$, as a function of viscosity ratio for two-dimensional irrotational flow $(\alpha=1)$. Data from the present study are compared with the observations of Taylor (1934), Rumscheidt \& Mason (1961), and Grace (1971). The unbroken curve shows the predictions of the $O\left(\epsilon^{2}\right)$ small-deformation theory discussed in $\$ 4.1$, and the dashed line shows the predictions of the largedeformation theory described in $\$ 4.2$. The result of Rallison's (1981) numerical calculation for $\lambda=1$ is also included.

As can be seen, all of the experimental data are in reasonable agreement, with the possible exception of the data point at $\lambda=1$ reported by Taylor (1934). Typical error bars for our experiments are shown on the figure. Uncertainties for the earlier experiments would presumably be larger owing to the difficulties associated with hand control of the flow device. For $\lambda \geqslant 0.05$, agreement between the burst predictions of the $O\left(\epsilon^{2}\right)$ small deformation theory and our observations is surprisingly good, especially considering the approximations inherent in the theory. The deviation between the theory and experiment is maximum near $\lambda=1.0$, with the theory underestimating $\mathbb{C}_{\mathrm{c}}$ by about $20 \%$. It is not known why this maximum occurs at $\lambda=1.0$. The numerical calculation of Rallison (1981) for $\lambda=1$ agrees very well with 


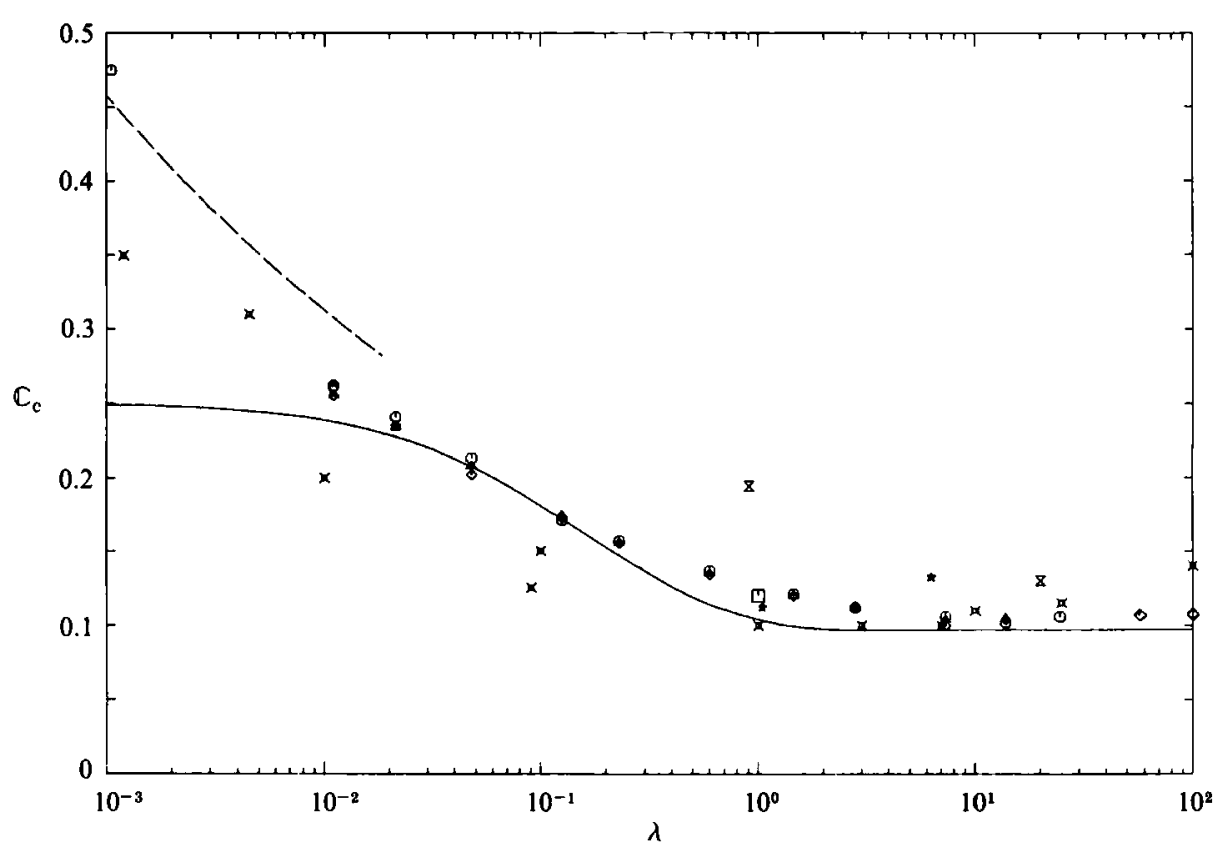

FigURe 25. Critical capillary number for burst as a function of viscosity ratio for $\alpha=1.0$. $\odot, \triangle$, $\diamond$, this work; $₫$, Grace (1971); $\star$, Rumscheidt \& Mason (1961); $\varangle$, Taylor (1934); $\square$, Rallison (1981) numerical;,$- O\left(\epsilon^{2}\right)$ small-deformation theory; ---, large-deformation theory.

the data. Further, for low viscosity ratios, the large-deformation theory predicts burst with acceptable accuracy.

The trend in figure 25 for large viscosity ratio is also of some interest. In our experiments, we see that the critical capillary number is essentially constant for viscosity ratios above about 3.0. In contrast, Grace (1971) concluded from his data that $\mathbb{C}_{\mathrm{c}}$ goes through a minimum for $\lambda$ about 1 , and then steadily increases with increase of the viscosity ratio above that value. We believe that Grace's conclusion is in error for the following reason: as the viscosity of the drop increases, the timescale for deformation increases as well. This makes the experiments more difficult since a much longer wait between increases in the shear rate must be allowed for the drop to attain a steady shape. In our experiment, the drop's position was controlled automatically, so great patience was possible. In a hand-controlled experiment, however, the difficulty in controlling the drop may have forced the experimenter to increase the shear rate before the drop attained an equilibrium shape (or burst) at the lower shear rate. The effect of such an error would be to observe drop burst at a higher shear rate than the actual critical value. The error would be expected to be greater for larger viscosity ratio since the time constant is higher for more viscous drops. The predictions of the $O\left(\epsilon^{2}\right)$ small-deformation theory (shown in figure 25) and the numerical calculations of Rallison \& Acrivos (1978) for axisymmetric extensional flow both support our conclusion that the critical capillary number for drop burst in irrotational flows tends to some constant as viscosity ratio increases.

Figure 26 shows the maximum steady deformation attained by the drop for pure extensional flow, $\alpha=1$ (as measured by the deformation parameter $D_{\mathrm{f}}$ ) at a capillary number just below the critical value for breakup. Data and theoretical predictions are presented from the same sources as for $\mathbb{C}_{\mathrm{c}}$. Accurate determination of $D_{\mathrm{f}, \mathrm{c}}$ is difficult, since the deformation changes rapidly with small increases in $\mathbb{C}$ near the 


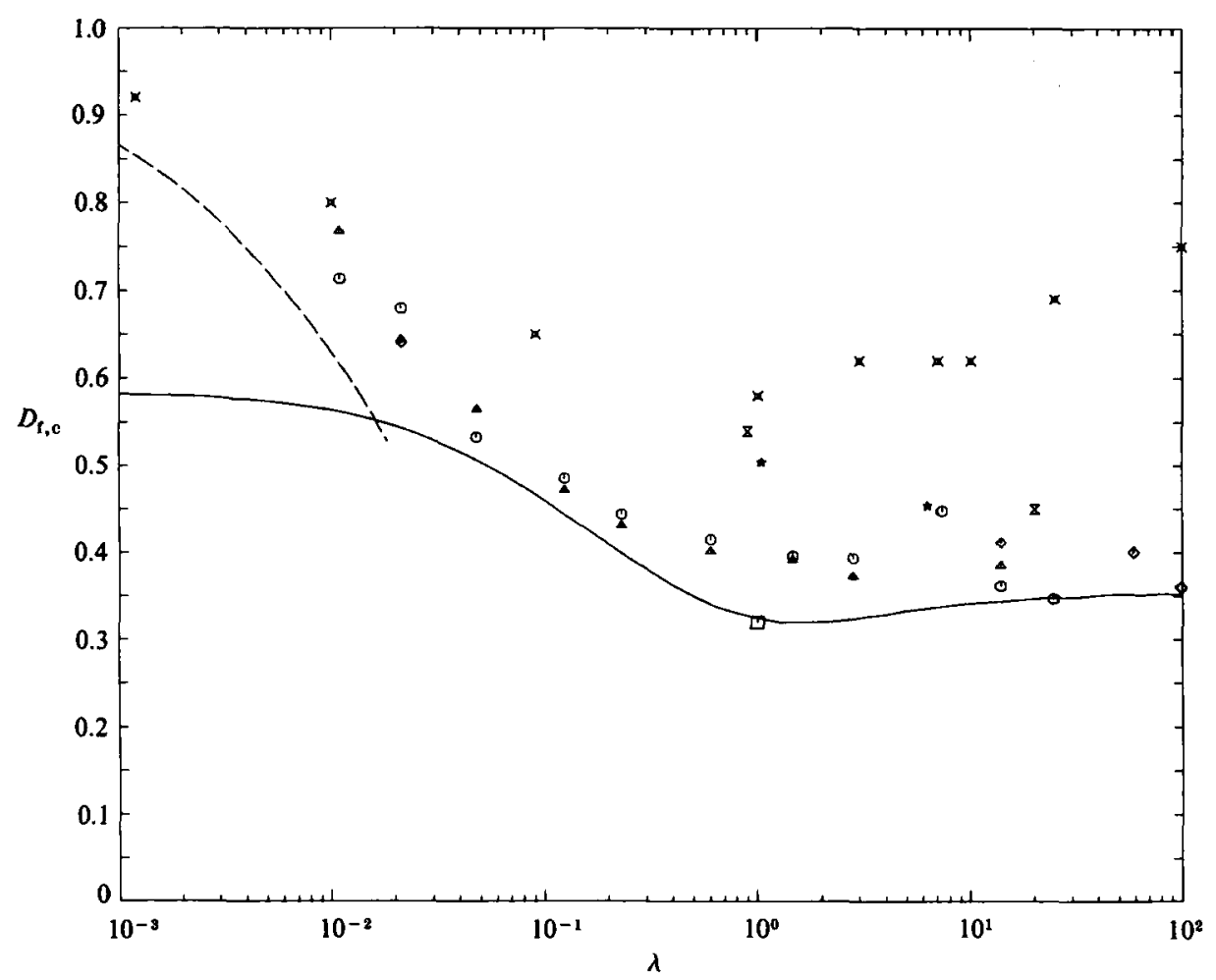

FIGURE 26. Deformation at critical capillary number as a function of viscosity ratio for $\alpha=1.0$. See figure 25 for key.

burst point (see figure 12, for example). This is reflected in the considerable scatter in the data. Agreement between our observations and the predictions of both small-deformation and large-deformation theories is acceptable. Most of the other available data indicates deformations at the bursting point which are considerably higher than we observed. In particular, the critical deformations reported by Grace (1971) are much higher than we observed. We attribute this discrepancy to the difficulty in distinguishing stable shapes from slowly evolving transients as discussed above.

Figure 27 shows photographs of the most extended stable shapes observed in irrotational flow $(\alpha=1)$ for each viscosity ratio investigated. For the lowest two viscosity ratios investigated, the shapes are highly extended and show pointed ends. With increasing viscosity ratio, the shapes become blunter. It is interesting that the shapes are nearly indistinguishable for all viscosity ratios greater than unity.

Figure 28 shows our observations for $\mathbb{C}_{\mathrm{c}}$ for all five flow types investigated. In the plots, the solid curves represent the predictions of the $O\left(\epsilon^{2}\right)$ small-deformation theory, the broken curves represent the predictions from our ad hoc generalization of the large-deformation theory, and the solid points show the results of Rallison's (1981) numerical calculation for $\lambda=1$. For all flow types except irrotational flow ( $\alpha=1$, for which comparisons to the results of other researchers has been presented above), there are no previous experimental studies to compare our results with.

It can be seen that agreement between the small-deformation theory and the observations is quite good for $\lambda \geqslant 0.05$ for all flow types. As was the case for $\alpha=1$, the small-deformation theory underestimates $\mathbb{C}_{\mathrm{c}}$ by approximately $20 \%$ for a viscosity 


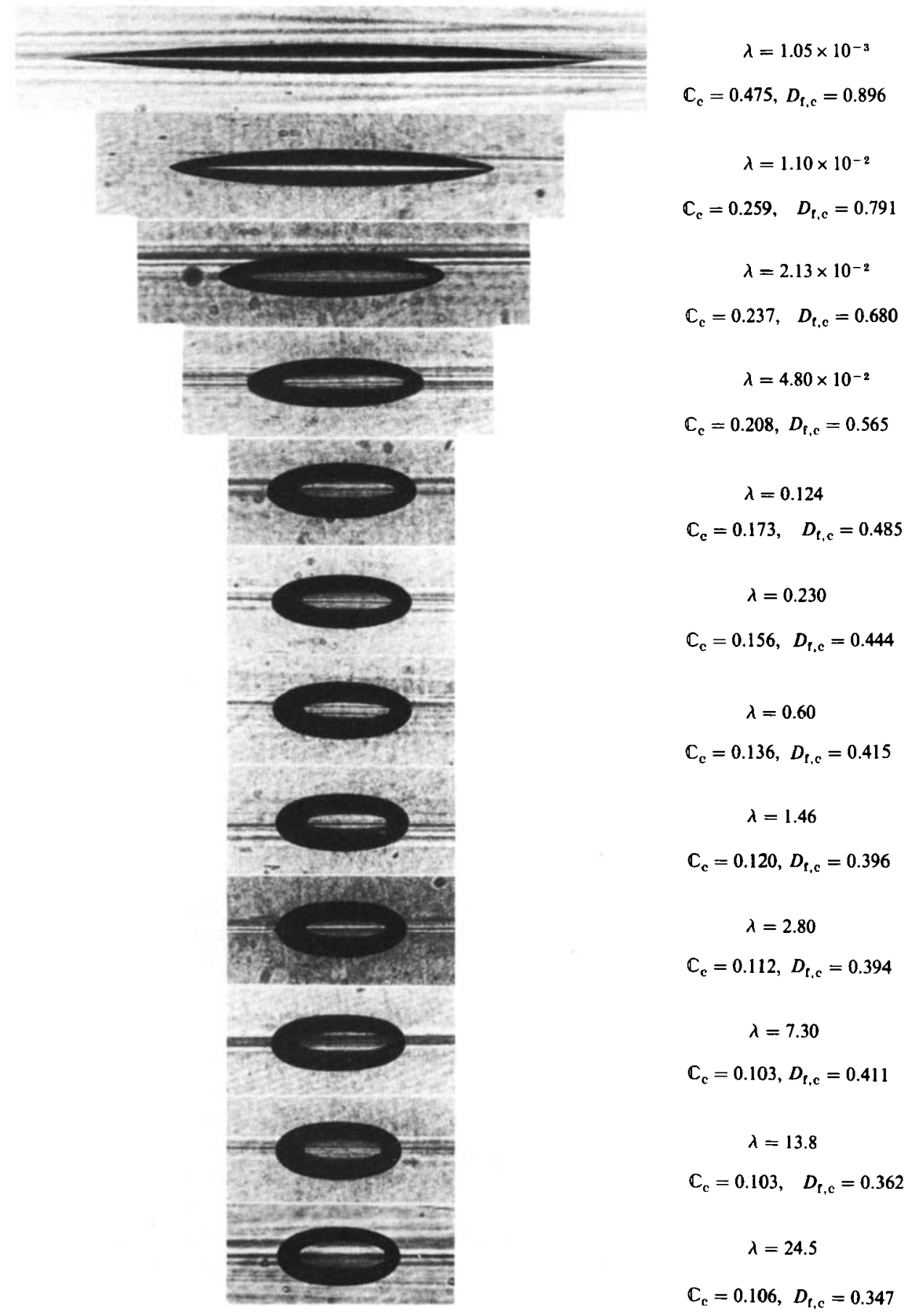

Fiaure 27. Drop shapes at critical capillary number for irrotational shear flow. 


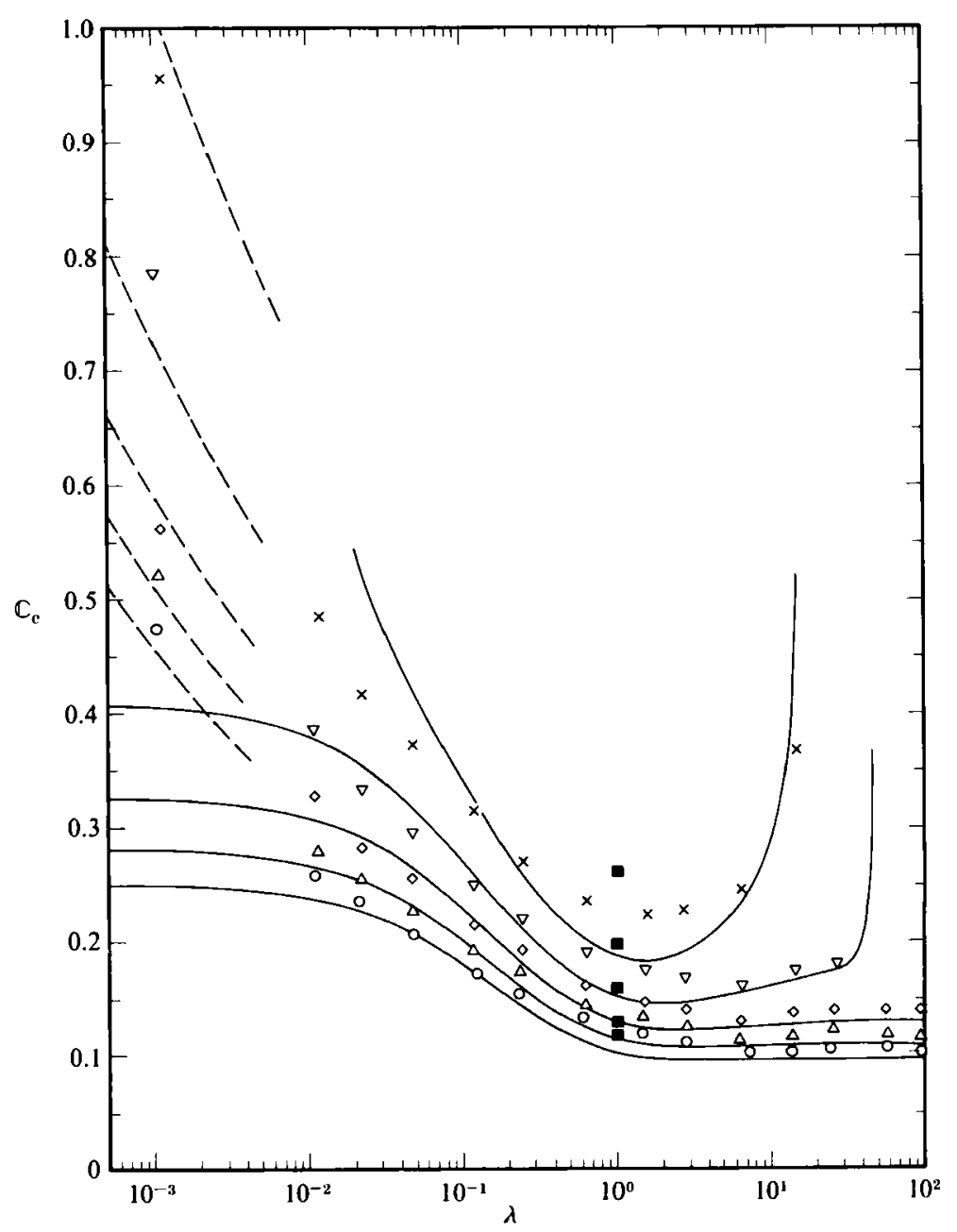

Figure 28. Effects of flow type on critical capillary number for drop burst. Plots shown are for $\alpha=1.0,0.8,0.6,0.4$ and 0.2 and go in order of increasing $\alpha$ from top to bottom. $0, \alpha=1.0 ; \triangle$, $\alpha=0.8 ; \diamond, \alpha=0.6 ; \nabla, \alpha=0.4 ; \times, \alpha=0.2 ; \square$, Rallison (1981) numerical; -,$O\left(\epsilon^{2}\right)$ smalldeformation theory; ---, large-deformation theory.

ratio near unity, while Rallison's (1981) numerical results provide a somewhat better estimate. For $\alpha=0.4$ and $\alpha=0.2$ the $O\left(\epsilon^{2}\right)$ small-deformation theory predicts a viscosity ratio (46.6 for $\alpha=0.4$ and 14.7 for $\alpha=0.2$ ) above which no drop burst is possible. This is analogous to the well-known behaviour in simple shear flow, except that the limiting viscosity ratio is lower $(\lambda=3.6)$ in simple shear flow, since the vorticity is higher. As mentioned above, the existence of viscosity ratios for which breakup is impossible for these two flows was verified experimentally. Drops with viscosity ratios of 27 and above could not be burst in flows with $\alpha=0.2$, and drops with $\lambda>57$ could not be burst in the flow type corresponding to $\alpha=0.4$.

For $\alpha=0.2$, the small-deformation theory also predicts a lower limit in viscosity ratio below which drop burst is not possible. However, this feature of the smalldeformation theory is not realistic. It should be noted that the predicted deformation at the point of burst becomes very large as the viscosity ratio decreases, and the small-deformation theory is far outside its expected region of validity. 


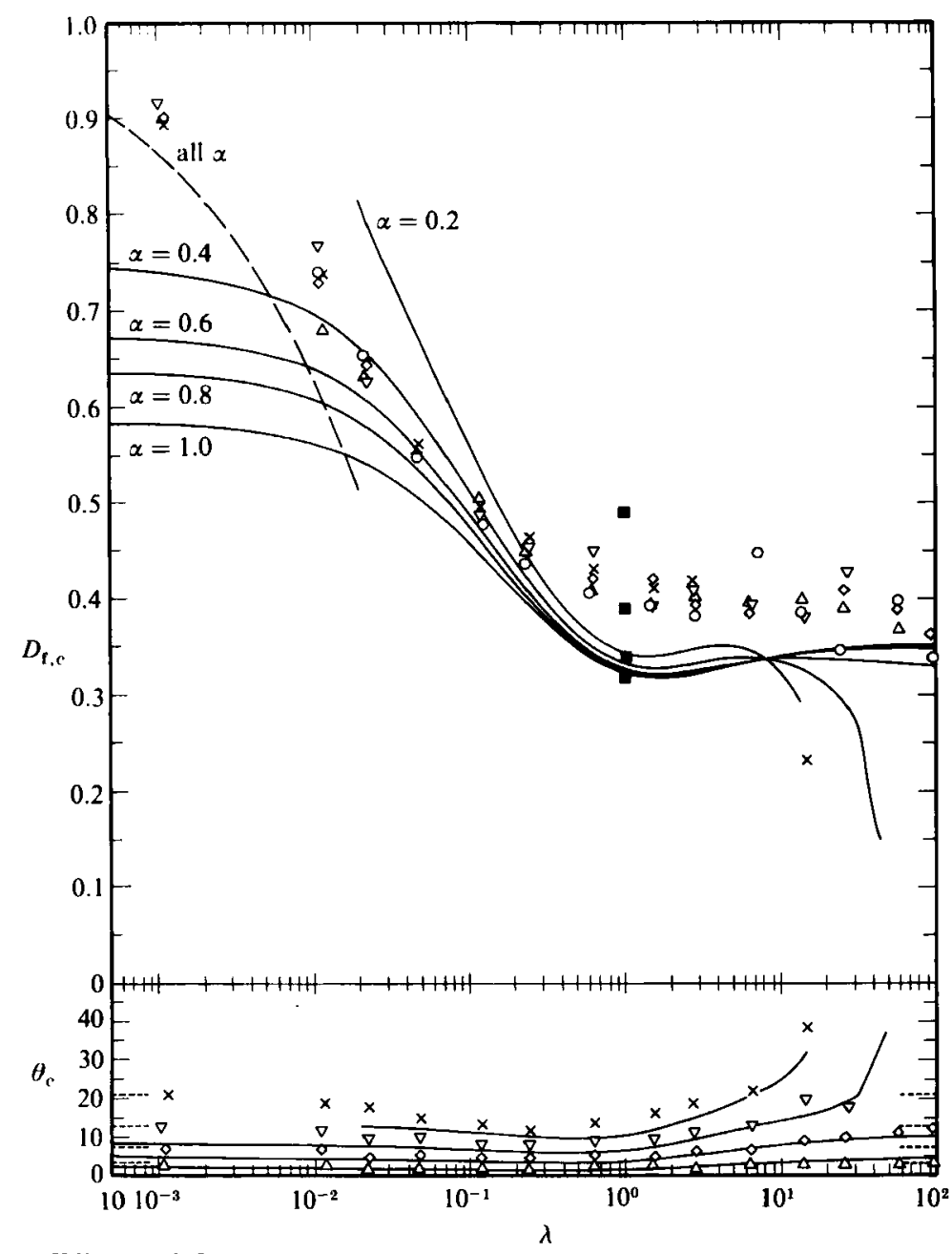

Figure 29. Effects of flow type on deformation and orientation angle at the critical capillary number. Numerical results of Rallison (1981) go in order of increasing $\alpha$ from top to bottom. Points for $\alpha=1.0$ and 0.8 coincide. See figure 28 for key.

While there is some scatter in the data, it appears from figure 28 that the ad hoc extension of the slender-body theory for $\alpha=1$ provides acceptable predictions for $\mathbb{C}_{c}$ in the absence of a more rigorous theory for intermediate flows, i.e. $\alpha<1$. It is probable that the agreement would be better if the experiments were extended to lower viscosity ratios.

Figure 29 shows the comparison between our results and the predictions of the available theories for the deformation, $D_{\mathrm{f}, \mathrm{c}}$, and orientation, $\theta_{\mathrm{c}}$, at the critical capillary number as a function of $\lambda$ for all flow types investigated. The largedeformation theory provides no prediction for $\theta_{\mathrm{c}}$ since it is assumed that the drops align with the angle of the exit streamline, an assumption which our observations support. Similarly, the $D_{\mathrm{f} . \mathrm{c}}$ predicted by the large-deformation theory is the same for all flow types, and is in reasonable agreement with the data for all flow types for the lowest-viscosity-ratio case investigated.

Generally, the agreement between predictions of the $O\left(\epsilon^{2}\right)$ small-deformation theory and the observations for $D_{\mathrm{f}, \mathrm{c}}$ are not as good as the agreement for $\mathbb{C}_{\mathrm{c}}$, most 
probably owing to the difficulty in accurately determining $D_{\mathrm{f}, \mathrm{c}}$. Similarly, the comparison between the numerical results and the experiments is also less favourable, but it should be noted that accurate numerical determination of $D_{\mathrm{f}, \mathrm{c}}$ is likewise difficult. One interesting feature of figure 29 is that both the small-deformation theory and the numerical results predict that the critical deformation should depend on the flow type, with both predicting larger $D_{\mathrm{f}, \mathrm{c}}$ for more rotational flows for $\lambda \leqslant 8$. (In figure 29, the solid rectangular points showing the numerical results for $D_{\mathrm{f}, \mathrm{c}}$ coincide for $\alpha=1.0$ and 0.8 , and go in decreasing order of $\alpha$ from bottom to top for the other three points.) In contrast, our experiments do not show any clear differences between the different flow types (except as discussed below for high viscosity ratios with $\alpha=0.2$ ). The reason for this discrepancy is not known, other than the large uncertainty in $D_{\mathrm{f}, \mathrm{c}}$.

\subsection{Discussion}

We have seen in the preceding two sections that the small-deformation and slender-drop theories provide predictions for deformation, critical flow strength, critical deformation, and critical orientation which are at times surprisingly accurate. In this section we present a more physical interpretation of our results, particularly in regard to the effect of vorticity on the deformation and burst process. We shall see that many of the interesting trends observed in the experiments can be explained through a qualitative analysis of the interaction between the orientation of the drop and the magnitude of the extension rate component which it experiences as a consequence of its orientation.

We first consider the orientation of a freely suspended body in a linear shear field of the type described by (5). Analytic results are readily available for the case where the body is a solid ellipsoid of revolution, but when the body is a deformable drop the situation is considerably more complicated. Thus our approach is to consider the orientation of a solid particle, and use the insight gained from this to make qualitative predictions for a deformable drop.

Since the creeping-flow equations and boundary conditions are linear for a suspended solid particle, we can consider separately the angular velocity of the solid particle in a purely rotational flow and in a purely extensional flow, and then superimpose the results to determine the angular velocity in the actual flow, which is a superposition of pure strain and vorticity. In the purely rotational flow, a particle of any shape rotates with the local fluid vorticity. In the flow of equation (5), the angular velocity due to the rotational component of the flow field is thus aligned with the $z$-axis, and is given by:

$$
\omega_{v}=-G(1-\alpha) .
$$

In the two-dimensional pure-straining flow, the angular velocity depends on the orientation and shape of the solid particle. When the particle is isotropic, the angular velocity is zero. When the shape is anisotropic (e.g. an ellipsoid of revolution), the angular velocity is greatest when the longest axis of the particle is aligned at $\pm 45^{\circ}$ from the principal axis of strain, since at this orientation the long axis is perpendicular to every streamline. When the long axis of the particle is aligned with the principal axis of strain, the angular velocity is zero. An expression for the angular velocity of an ellipsoid of revolution in a two-dimensional pure-straining flow $(u=g x, v=-g y, z=0)$ is given by Chaffey, Takano \& Mason (1965):

$$
\omega_{\mathrm{e}}=-g b \sin 2 \theta,
$$


where $b$ is a scalar characterizing the eccentricity of the particle. It is related to the equivalent axis ratio of an ellipsoid $r_{\mathrm{e}}$ by

$$
b=\frac{\left(r_{\mathrm{e}}^{2}-1\right)}{\left(r_{\mathrm{e}}^{2}+1\right)} .
$$

This quantity ranges from zero for a sphere to +1 for an infinite rod. In (38) $\theta$ is the angle between the longest axis of the particle and the $x$-axis. In the flow of (5), the strength of the extensional portion of the flow (i.e. $g$ in $(38)$ ) is given by $G(1+\alpha)$.

Superimposing the angular velocities in the rotational and extensional flows then gives the angular velocity in the actual flow:

$$
\omega=\omega_{v}+\omega_{\mathrm{e}}=-G(1-\alpha)-G(1+\alpha) b \sin 2 \theta .
$$

To find the steady orientations, we set the angular velocity equal to zero and solve:

$$
\theta=\frac{1}{2} \sin ^{-1}\left[\frac{\alpha-1}{b(\alpha+1)}\right]
$$

This equation has no solutions for small $b$ (unless $\alpha=1$, a vorticity-free flow), which means that nearly spherical solid particles will always rotate in a flow with vorticity. For $b \geqslant(\alpha-1) /(\alpha+1)$, solutions exist, with particle alignment depending on $b$ and $\alpha$. The least eccentric particle which has a steady orientation, $b=(\alpha-1) /(\alpha+1)$, is aligned at $-45^{\circ}$. As $b$ increases (increasing eccentricity) the magnitude of the orientation angle decreases. An infinite $\operatorname{rod}(b=1)$ aligns with the exit streamline of the flow field, $\theta=\frac{1}{2} \sin ^{-1}[(\alpha-1) /(\alpha+1)]$. For a solid particle, no steady orientations with the magnitude of $\theta$ less than this value are possible. Thus, if we did a series of experiments with solid ellipsoids of increasing axis ratio, we should find a monotonic change in the orientation angle $\theta$ from an initial value $-45^{\circ}$ for a 'slightly deformed' particle to $\theta=\frac{1}{2} \sin ^{-1}[(\alpha-1) /(\alpha+1)]$ corresponding to the exit streamline of the flow for a highly elongated particle.

The orientation of viscous drops is much more complicated since drops can accommodate viscous stresses through deformation. Therefore, there are two timescales of importance. The first is the timescale of the flow given by $G^{-1}$. This timescale characterizes the strength of the vorticity which is tending to rotate the drop. It appears in the solid-particle case as well, but since it is the only timescale, it does not affect the equilibrium orientation in that case. The second timescale characterizes the response of the drop to viscous stresses, and is given by $\lambda \mu a / \sigma$. For a deformable drop, the ratio of the second timescale to the first, $C \lambda$, is an additional parameter affecting the equilibrium drop orientation. The other two parameters (which affect the orientation of a solid particle as well) are the flow type and the anisotropy (or degree of non-sphericity) of the drop or particle. Unfortunately, these three parameters are not independent because the deformation of the drop is related to the timescale ratio (through both $C$ and $\lambda$ ) and to the flow type. Thus it is difficult to determine the effect of each parameter separately, and it is necessary to consider increases of the timescale ratio due to increasing viscosity ratio and increasing capillary number separately.

When the deformation timescale is short compared to the flow timescale (low $C \lambda$ ), the drop can be thought of as responding instantaneously to the deforming viscous stresses. Since the extensional portion of the flow field is responsible for these deforming stresses (cf. the $O(1)$ terms in the small deformation theory of equation (13)), it follows that when $C \lambda$ is small, drops will be aligned with the principal axis 
of strain (the $x$-axis in our experiments). This may be contrasted with slightly deformed solid particles where the particle initially aligns, with increase of its axis ratio, at $\theta=-45^{\circ}$ from the principal strain axis. In the experiments, we observed alignment with the $x$-axis for all viscosity ratios and flow types when the capillary number was sufficiently low. When $\lambda$ was small and $\mathbb{C}$ moderate, the deformation became too large for the drops to respond instantaneously, and they were rotated away from the $x$-axis.

An illustration of the effect of increasing the timescale ratio solely through increases in the deformation timescale is provided by comparing the orientation of drops at fixed values of $\mathbb{C}$ and $\alpha$ in the series of plots in figures 16 to 23 . As the ratio of drop to suspending fluid viscosity increases, more solid-like behaviour is observed, with the more viscous drops in the series exhibiting orientation angles closer to the $-45^{\circ}$ expected for slightly deformed solid particles.

When the timescale ratio $C \lambda$ is instead increased by decreasing the flow timescale (increasing $G$ and thus $\mathbb{C}$ at fixed $\lambda$, as is done in individual experiments), the situation is more complicated because increasing $\mathbb{C}$ also causes greater deformation. From (41) above, it is clear that the effect of this greater deformation is to align the drop more closely with the exit streamline of the flow. For low viscosity ratios the orientation of the drops is between the $x$-axis and the exit streamline. Thus when $C$ is increased, both the increase in $C \lambda$ and the consequential increase in deformation serve to rotate the drop towards the exit streamline, and the result is a monotonic increase in the magnitude of the orientation angle with increasing $C$.

In contrast, when $\lambda$ is greater than about 3.0, the magnitude of the orientation angle may actually exceed that of the exit streamline for some values of $\mathbb{C}$. In that case, further increases in $\mathbb{C}$ have two competing effects. The effect of increasing the time constant ratio is to rotate the drop further from the extensional axis. However, the consequential increase in deformation acts to align the drop closer to the exit streamline of the flow, thus tending to decrease the magnitude of the orientation angle. The net result of these competing effects is the appearance of a maximum in the magnitude of the orientation angle as the shear rate is increased in some high-viscosity-ratio experiments. This is illustrated in figures 20 to 23 . Note that the $O\left(\epsilon^{2}\right)$ small-deformation theory predicts this behaviour quite well.

The orientation of a drop has a strong bearing on the effective strain rate which it experiences in the flow. We define effective strain rate as the constant of proportionality between the component of fluid velocity (in the undisturbed flow field) parallel to the longest axis of the drop and the displacement along that axis. When $\boldsymbol{x}$ is a unit vector in the direction of the orientation of the drop axis, this is given by $\boldsymbol{x} \cdot \boldsymbol{u}$. Since the flow is a linear shear field, $\boldsymbol{u}=\boldsymbol{\nabla} \boldsymbol{u} \cdot \boldsymbol{x}$, and substitution of (5) gives:

$$
\text { effective strain rate }=G\left(\frac{1+\alpha}{2}\right) \cos 2 \theta .
$$

Thus, the effective strain rate is a strong function of orientation, ranging from a maximum of $G(1+\alpha) / 2$ when the drop is aligned with the principal axis of strain (the $x$-axis in our device), to zero when the drop is aligned at $-45^{\circ}$. Note that when the drop is aligned with the exit streamline $(\sin 2 \theta=(\alpha-1) /(\alpha+1))$, the effective strain rate is $G \alpha^{\frac{1}{2}}$.

A physical understanding of many of the trends observed in our drop-burst experiments can be obtained by considering the interaction between the drop orientation in the flow and the effective strain rate it experiences as a consequence of that orientation. For example, in slightly rotational flows $(\alpha=0.8$ and 0.6$)$ the 
capillary number required for burst of intermediate- and high-viscosity-ratio drops (for which $\mathbb{C}_{\mathrm{c}}$ and $D_{\mathrm{f}, \mathrm{c}}$ are nearly constant for irrotational flow as shown in figures 25 and 26) is correlated with the orientation angle near the burst point. From figure 29 , we note that drops of intermediate viscosity ratio are apparently of low enough viscosity to allow the dissipation of vorticity through internal circulation, so their orientation at burst is between the principal axis of strain and the exit streamline of the flow. With increasing viscosity ratio, however, the timescale for deformation increases, and the drops tend towards more solid-like behaviour, becoming oriented further from the principal axis of strain, with the magnitude of the orientation angle even exceeding that of the exit streamline of the flow. As the magnitude of the orientation angle increases, the effective strain rate at a given shear rate diminishes, and hence a larger shear rate is required for burst. This explains the appearance of a minimum in $\mathbb{C}_{\mathrm{c}}$ at a viscosity ratio of about 5.0 in figure 28 .

In more rotational flows, more dramatic effects are observed. For example, in flows with $\alpha \leqslant 0.4$, the small-deformation theory predicts (and our experiments confirm) the existence of limiting viscosity ratios, above which burst is impossible. This is evidently because the orientation of the drop is such that the effective strain rate is very low. For example, in a simple shear flow, drops of viscosity ratio 3.5 or larger align very close to $-45^{\circ}$, where the effective strain is nearly zero. Increases in the shear rate increase the timescale ratio $\mathbb{C} \lambda$, causing the drops to align still closer to the $-45^{\circ}$ line, neutralizing the effect of the increased flow strength, and making drop burst impossible. This same phenomena occurs for $\alpha=0.2$ and 0.4 as well, but at a higher viscosity ratio since the ratio of vorticity to strain is lower.

Similar reasoning can also explain the interesting trends predicted for $D_{\mathrm{f}, \mathrm{c}}$ and $\theta_{\mathrm{e}}$ for high-viscosity-ratio drops in flows with $\alpha=0.4$ and 0.2 . The small-deformation theory predicts that as the viscosity ratio increases above about 10 , the deformation at the bursting point decreases, and the magnitude of the orientation angle increases. This is caused by an interaction between the deformation and orientation. As we have noted above, high-viscosity-ratio drops are rotated by the vorticity such that the magnitude of the orientation angle is greater than that of the exit streamline. Near the bursting point, when the shear rate is increased slightly, the drop elongates in response, and the greater eccentricity causes it to be rotated towards the exit streamline of the flow, where the effective strain is larger. This higher effective strain rate causes greater elongation, further decrease in the magnitude of the orientation angle, still higher effective strain rate, etc., and leads to drop burst. The maximum stable deformation, of course, is the deformation at which this process is initiated, and this apparently decreases with increasing viscosity ratio. Our data reflect this trend for $\alpha=0.2$, but the data point for $\lambda=27$ indicates an opposite trend for $\alpha=0.4$, possibly due to experimental error in the (difficult) determination of $D_{\mathrm{p}, \mathrm{c}}$. This trend for high viscosity ratios is not observed for simple shear flow, since in that case the magnitude of the orientation angle is always less than that of the exit streamline $\left(\theta_{\mathrm{e}}=-45^{\circ}\right)$ and the interaction described above cannot occur.

\section{Conclusions}

\subsection{Small-deformation theory}

The small-deformation theory of Barthès-Biesel \& Acrivos (1973a) gives adequate predictions for drop deformation for $\lambda \geqslant 0.05$. The $O\left(\epsilon^{2}\right)$ version of the theory generally give better predictions for the deformation than does the $O(\epsilon)$ theory. It should be noted that good agreement between the experimental and theoretical scalar 
deformation measure, $D_{\mathrm{f}}$, is sometimes attained even when the calculated shape is quite different from the observed drop shape. The $O\left(\epsilon^{2}\right)$ theory always gives better predictions for the critical capillary number at which drop burst oceurs than does the $O(\epsilon)$ theory. The $O\left(\epsilon^{2}\right)$ theory predicts the qualitative features of drop burst with surprising accuracy for viscosity ratios greater than about 0.05 . The agreement is for all flow types investigated in our experiments and for simple shear flow. In particular, the theory correctly predicts a limiting viscosity ratio above which drop burst is impossible for $\alpha=0.4,0.2$, and 0.0 . It can be used for quantitative estimates of $C_{c}$, accurate to within about $30 \%$, for the two-dimensional flows we considered when $\lambda>0.05$.

\subsection{Large-deformation theory}

The large-deformation theory of Hinch \& Acrivos (1979) gives adequate predictions for drop shape and burst for $\lambda<0.01$ for two-dimensional pure straining motion $(\alpha=1)$, and our ad hoc extension for other flow fields provides an acceptable result for intermediate flows in the absence of a more rigorous theory. In our experiments, the lowest viscosity ratio considered was $\lambda=0.001$, and in that case the predictions for $C_{\mathrm{c}}$ and $L / a$ at burst were accurate to within about $15 \%$ for all $\alpha$. It is expected that the agreement would improve for lower $\lambda$, since the slenderness of the drop increases with decreasing $\lambda$.

\subsection{Numerical results}

The numerical calculations of Rallison (1981) for $\lambda=1$ are in good agreement with the data for $\mathbb{C}_{c}$. The predicted critical deformations do not agree nearly so well with our observations. This can be attributed to the difficulties in accurately determining the critical deformation, both experimentally and numerically. Numerical results for other viscosity ratios are not yet available for the flows we studied.

The authors with to thank Howard Stone for performing several of the more difficult experiments, and Professor Andreas Acrivos for helpful discussions during his sabattical year at Caltech. One of the authors (B.J.B.) was partially supported through a National Science Foundation Graduate Fellowship. This work was supported by a grant from the Fluid Mechanics program of the National Science Foundation.

\section{REFERENCES}

Acrivos, A. 1983 The breakup of small drops and bubbles in shear flows. Ann. N.Y. Acad. Sci. 404, 1-11.

Acrivos, A. \& Lo, T. S. 1978 Deformation and breakup of a single slender drop in an extensional flow. J. Fluid Mech. 86, 641-672.

Barthès-Biesel, D. \& Acrivos, A. $1973 a$ Deformation and burst of a liquid droplet freely suspended in a linear shear field. J. Fluid Mech. 61, 1-21.

Barthès-Biesel, D. \& Acrivos, A. $1973 b$ The rheology of suspensions and its relation to phenomenological theories for non-Newtonian fluids. Intl J. Multiphase Flow 1, 1-24.

Bentley, B. J. 1985 Drop deformation and burst in two-dimensional flows. Ph.D. thesis, California Institute of Technology.

Bentley, B. J. \& Leal, L. G. 1986 A computer-controlled four-roll mill for investigations of particle and drop dynamics in two-dimensional linear shear flows. J. Fluid Mech. 167, 219-240.

Buckmaster, J. D. 1972 Pointed bubbles in slow viscous flow. J. Fluid Mech. 55, 385-400.

Buckmaster, J. D. 1973 The bursting of pointed drops in slow viscous flow. Trans. ASME E: J. Appl. Mech. 40, 18-24.

Chaffey, C. E., Takano, M. \& Mason, S. G. 1965 Particle motions in sheared suspensions. 16. Orientations of rods and disks in hyperbolic and other flows. Can. J. Phys. 43, 1269-1287. 
Cox, R. G. 1969 The deformation of a drop in a general time-dependent fluid flow. J. Fluid Mech. $37,601-623$.

Frankel, N. A. \& Acrivos, A. 1970 The constitutive equation for a dilute emulsion. J. Fluid Mech. 44, 65-78.

Fulder, G. G. \& Leal, L. G. 1981 Flow birefringence of concentrated polymer solutions in two-dimensional flows. J. Polymer Sci. Polymer Phys. Ed. 19, 557-587.

GIESEKUS, H. 1962 Strömungen mit konstantem Geschwindigkeitsgradienten und die Bewegung von dar in suspendierten Teilchen. Teil II : Ebene Strömungen und eine experimentelle Anordnung zu ihrer Realisierung. Rheol. Acta. 2, 113-121.

Grace, H. P. 1971 Dispersion phenomena in high viscosity immiscible fluid systems and application of static mixers as dispersion devices in such systems. Eng. Found. Res. Conf. Mixing, 3rd, Andover, N.H. Republished 1982 in Chem. Engng Commun. 14, 225-277.

HAKIMI, F. S. \& SCHOWALTER, W. R. 1980 The effects of shear and vorticity on deformation of a drop. J. Fluid Mech. 98, 635-645.

Hinch, E. J. \& ACRIvos, A. 1979 Steady long slender droplets in two-dimensional straining motion. J. Fluid Mech. 91, 401-414.

Hinch, E. J. \& ACRivos, A. 1980 Long slender drops in a simple shear flow. J. Fluid Mech. 98, $305-328$.

Rallison, J. M. 1980 A note on the time-dependent deformation of a viscous drop which is almost spherical. J. Fluid Mech. 98, 625-633.

RALLISON, J. M. 1981 A numerical study of the deformation and burst of a viscous drop in general shear flows. J. Fluid Mech. 109, 465-482.

Rallison, J. M. 1984 The deformation of small viscous drops and bubbles in shear flows. Ann. Rev. Fluid Mech. 16, 45-66.

Rallison, J. M. \& ACrivos, A. 1978 A numerical study of the deformation and burst of a viscous drop in an extensional flow. J. Fluid Mech. 89, 191-209.

Rumscheidt, F. D. \& Mason, S. G. 1961 Particle motions in sheared suspensions. 12. Deformation and burst of fluid drops in shear and hyperbolic flows. J. Colloid Interface Sci. 16, 238-261.

TAYlor, G. I. 1932 The viscosity of a fluid containing small drops of another fluid. Proc. R. Soc. Lond. A 138, 41-48.

TAYLOR, G. I. 1934 The formation of emulsions in definable fields of flow. Proc. R. Soc. Lond. A $146,501-523$.

TaYlor, G. I. 1964 Conical free surfaces and fluid interfaces. Proc. Intl Congr. Appl. Mech. 11th, Munich, pp. 790-796.

Torza, S., Cox, R. G. \& Mason, S. G. 1972 Particle motions in sheared suspension. 27. Transient and steady deformation and burst of liquid drops. J. Colloid Interface Sci. 38, 395-411.

YoungREN, G. K. \& ACRIvos, A. 1976 On the shape of a gas bubble in a viscous extensional flow. J. Fluid Mech. 76, 433-442. 